\title{
Handcuffing intrinsically disordered regions in Mlh1-Pms1 disrupts mismatch repair
}

\author{
Christopher M. Furman ${ }^{1}$, Ting-Yi Wang ${ }^{2}$, Qiuye Zhao ${ }^{2}$, Kumar Yugandhar ${ }^{2}$, Haiyuan $\mathrm{Yu}^{2}$ and \\ Eric Alani ${ }^{\oplus 1, *}$
}

${ }^{1}$ Department of Molecular Biology and Genetics, Cornell University, Ithaca, NY 14853, USA and ${ }^{2}$ Department of
Computational Biology, Cornell University, Ithaca, NY 14853, USA

Received March 10, 2021; Revised July 19, 2021; Editorial Decision July 27, 2021; Accepted July 28, 2021

\begin{abstract}
The DNA mismatch repair (MMR) factor Mlh1-Pms1 contains long intrinsically disordered regions (IDRs) whose exact functions remain elusive. We performed cross-linking mass spectrometry to identify interactions within Mlh1-Pms1 and used this information to insert FRB and FKBP dimerization domains into their IDRs. Baker's yeast strains bearing these constructs were grown with rapamycin to induce dimerization. A strain containing FRB and FKBP domains in the MIh1 IDR displayed a complete defect in MMR when grown with rapamycin. but removing rapamycin restored MMR functions. Strains in which FRB was inserted into the IDR of one MLH subunit and FKBP into the other subunit were also MMR defective. The MLH complex containing FRB and FKBP domains in the MIh1 IDR displayed a rapamycin-dependent defect in MIh1-Pms1 endonuclease activity. In contrast, linking the MIh1 and Pms1 IDRs through FRBFKBP dimerization inappropriately activated Mlh1Pms1 endonuclease activity. We conclude that dynamic and coordinated rearrangements of the MLH IDRs both positively and negatively regulate how the MLH complex acts in MMR. The application of the FRB-FKBP dimerization system to interrogate in vivo functions of a critical repair complex will be useful for probing IDRs in diverse enzymes and to probe transient loss of MMR on demand.
\end{abstract}

\section{INTRODUCTION}

Intrinsically disordered regions (IDRs), characterized as being conformationally flexible, are present in roughly $30 \%$ of eukaryotic proteins. While many IDRs have unknown roles, some facilitate interactions with other proteins or between regions of the same protein. IDRs are often critical for overall protein function, but the mechanisms of action are lacking because IDRs do not have a clear structure and amino acid substitutions in IDRs often have no effect (1-3). Here, we investigate the roles that the IDRs of the highly conserved MutL family proteins play during DNA mismatch repair (MMR).

MMR acts to correct DNA misincorporation errors that arise during replication (4). In baker's yeast, DNA mismatches are recognized by the MutS homolog (MSH) proteins Msh2-Msh6 and Msh2-Msh3. These proteins act as ATP-modulated sliding clamps to recruit MLH proteins, principally Mlh1-Pms1, which nick the newly replicated strand of DNA in the vicinity of the mismatch through steps that require interactions with the DNA polymerase processivity clamp PCNA (Figure 1A; 5-8). ATP binding by Mlh1-Pms1 stimulates its endonuclease activity on doublestranded DNA, and this stimulation is significantly greater on DNA substrates loaded with PCNA (9-13). Nicks located $5^{\prime}$ to the mismatch act as entry sites for Exo1, a $5^{\prime}$ to $3^{\prime}$ exonuclease, to digest the DNA strand containing the misincorporation error. The single stranded binding protein RPA then coats the ssDNA gap, after which DNA polymerase $\delta$ (and possibly DNA polymerase $\varepsilon ; 14$ ) resynthesizes the gapped DNA. A partially redundant Exo1independent mechanism requires Mlh1-Pms1 to make multiple nicks on the newly replicated strand in the vicinity of the mismatch, enabling DNA polymerase $\delta$ to remove the mismatch via DNA synthesis and strand displacement. The nicks generated by both pathways are sealed by DNA ligase $(9,15-18)$.

Single molecule studies have provided a mechanistic view of how Mlh1-Pms1 interacts with MSH proteins and mismatched DNA (Figure 1A). Studies by Gorman et al. $(6,19)$ showed that Mlh1-Pms1 is targeted to lesion-bound Msh2Msh6 by one-dimensional hopping and three-dimensional diffusion mechanisms, and the addition of ATP provoked the release of Msh2-Msh6/Mlh1-Pms1 from mismatched DNA. How do the MLH proteins accomplish these diffusion steps? Initial hints came from atomic force microscopy and ATP hydrolysis analyses which showed that ATP binding by Mlh1-Pms1 is accompanied by conformational rearrangements involving IDRs in both subunits (Figure 1B;

\footnotetext{
${ }^{*}$ To whom correspondence should be addressed. Tel: +1 607254 4811; Email: eea3@cornell.edu
} 

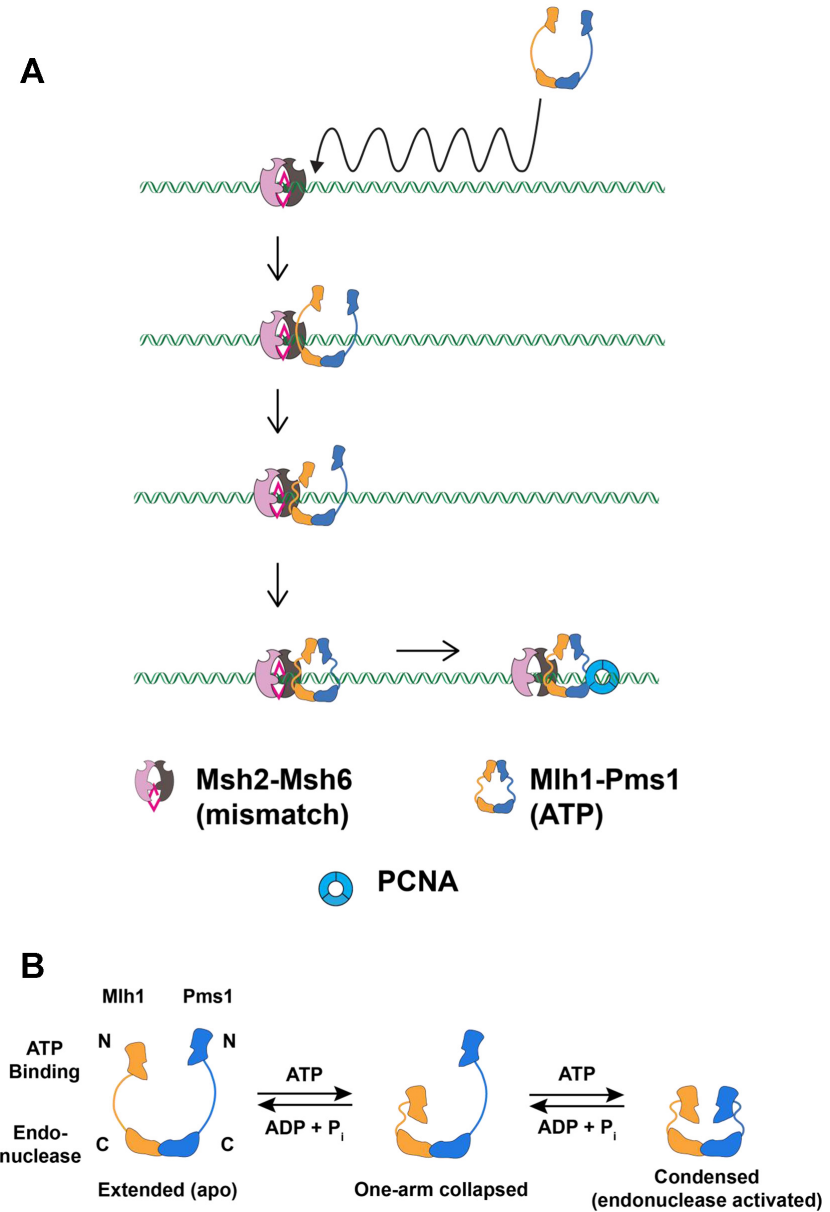

Figure 1. ATP-driven conformational changes in Mlh1-Pms1 during MMR. (A) A model for Mlh1-Pms1 interactions with MSH proteins during MMR. See Introduction for details. (B) Sacho et al. (21) proposed that ATP binding to one subunit of the MLH heterodimer promotes the formation of a one-armed collapsed state. Subsequent binding to the second subunit condenses its linker arm to yield a condensed complex that is thought to represent the activated endonuclease state.

20-22). Mlh1 (IDR is 164 amino acids in length) and Pms1 (IDR is 295 amino acids in length) each contain IDRs separated by globular N- and C-terminal domains (Supplementary Figure S1A; 20,23-26). Kim et al. (9) showed that an MMR defective Mlh1-Pms1 complex containing shorter IDRs retained wild-type DNA binding affinity but showed diffusion defects on both naked and nucleosomecoated DNA. They also showed that the IDRs regulated the ATP hydrolysis and nuclease activities encoded by the Nand C-terminal domains of the complex, respectively. These studies suggest multi-functional roles for IDRs in regulating Mlh1-Pms1 diffusion on DNA and nucleolytic processing. Liu et al. (27) showed that Escherichia coli MutS sliding clamps recruited MutL on mismatched DNA to form a MutS-MutL search complex. In their model they hypothesized that 'an open conformation of EcMutL is required to interact with EcMutS sliding clamps, which then binds ATP to form a second, exceedingly stable, ring-like clamp' that acts as a search complex $(27,28)$.
The above models argue that the MLH IDRs play key roles in facilitating these steps $(9,21,29)$, but it remains unclear how they occur. Sacho et al. (21) and Kunkel and Erie (4) proposed that ATP binding to the Mlh1 subunit of the Mlh1-Pms1 heterodimer promotes the formation of a one-armed collapsed complex in which the $\mathrm{N}$-terminal ATP binding domain of Mlh1 and its IDR fold into or near the C-terminal Mlh1-Pms1 domain (Figure 1B). ATP binding to Pms1 then condenses its linker arm to yield a condensed complex that positions the N-terminus of Mlh1 and Pms1 in proximity to the C-terminal domains. This conformational change, modulated through the IDR domains of both proteins, is hypothesized to change Mlh1-Pms1 affinity for DNA and activate its endonuclease activity located at the C-terminus of Pms1 (11,21,30-32).

We performed a direct test of the above models by inserting FRB and FKBP dimerization domains into the Mlh1 and Pms1 IDRs. The FRB and FKBP domains form tight dimers upon the addition of the small molecule rapamycin (33). Introducing both FRB and FKBP domains into the Mlh1 IDR conferred in the presence of rapamycin a null MMR phenotype. This condition also disrupted the endonuclease activity of Mlh1-Pms1, showing that changes in conformation in the Mlh1 IDR are critical for MMR. We also inserted FRB and FKBP domains into each IDR and showed that strains containing these constructs displayed strong MMR defects when grown in the presence of rapamycin. In vitro studies of one such complex showed that MMR defects were accompanied by the inappropriate activation of Mlh1-Pms1 and a defect in Mlh1-Pms1 binding to DNA. Our observations indicate that the MLH IDRs mediate dynamic conformational steps required for Mlh1Pms1 to form a clamp on DNA and for licensing subsequent repair steps. Importantly, we provide a general strategy to reversibly lock conformational changes in IDR domains that can be used to study a diverse number of cellular processes.

\section{MATERIALS AND METHODS}

\section{Media}

Saccharomyces cerevisiae strains were grown at $30^{\circ} \mathrm{C}$ in either yeast extract-peptone-dextrose (YPD) media or minimal selective media (SC; 34). When required, geneticin (Invitrogen, San Diego) was added at $200 \mu \mathrm{g} / \mathrm{ml}$ (35).

\section{Strains}

S288c background derived yeast strains are listed in Supplementary Table S1, with details regarding their construction available upon request. Briefly, the rapamycin resistant strain EAY4450, derived from EAY1269, was constructed as described by Zhu et al. (36) for the yeast strain JJY70. Plasmids bearing $M L H 1$ and PMS1 derivatives (Supplementary Table S2) were digested with the appropriate restriction enzymes and introduced into EAY4450 using methods described in Rose et al. (34) and Gietz et al. (37). $m l h 1 \Delta$ derivatives of EAY4450, EAY4488-4490, were constructed by digesting pEAI160 (mlh1 $\Delta::$ KanMX) with the $S p h \mathrm{I}$ and $K p n \mathrm{I}$ prior to transformation. All integrants were genotyped by PCR using primers that map outside of 
the restriction sites used for integration, and the presence of specific alleles was confirmed by DNA sequencing.

\section{Plasmids}

Plasmids used in this study are listed in Supplementary Table S2. Briefly, integration vectors (pEAA672, 674, 675, 713 derived from pEAA213-MLH1::KanMX; pEAI453, $454,455,468$, derived from pEAA238- $P M S 1$ ) containing $F R B$ or $F K B P$ insertions were constructed using $\mathrm{HiFi}$ Gibson cloning (New England Biolabs, Ipswich, MA), with PCR fragments generated from pEAA213 or pEAA238, and gBlocks (Integrated DNA Technologies, Coralville, IA) encoding FRB and FKBP protein domains $(23,38,39)$. The FRB and FKBP domains were inserted immediately after the indicated amino acid position of Mlh1 and Pms1 (Supplementary Figure S1A). PMS1 integration vectors were constructed through HiFi Gibson cloning by inserting the LEU2 gene from pRS415 (40) downstream of PMS1 in the ARS-CEN vectors pEAA671, 676, 677, and 678. MLH1 (pEAE 269, 446, 447, 448, 460) and PMS1 (pEAE431, 433, 435) protein expression vectors were derived from pMH1 (GAL1-MLH1-VMA-CBD,2 $\mu$, $T R P 1)$ and pMH8 (GAL10-PMS1,2 $\mu, L E U 2)$, respectively (41). The DNA sequence of the open reading frames (including $300 \mathrm{bp}$ upstream and $150 \mathrm{bp}$ downstream) of constructs was confirmed by Sanger DNA sequencing (Cornell BioResource Center). The amino acid sequence of the FRB insertions, with glycine $(\mathrm{G})$ and serine (S) linkers shown in bold is: GSILWHEMWHEGLEEASRLYFG ERNVKGMFEVLEPLHAMMERGPQTLKETSFNQ AYGRDLMEAQEWCRKYMKSGNVKDLLQAWDLY YHVFRRISKGS. The amino acid sequence of the FKBP insertions, with glycine and serine linkers shown in bold is: GSGVQVETISPGDGRTFPKRGQTCVVHYTGMLED GKKFDSSRDRNKPFKFMLGKQEVIRGWEEGVA QMSVGQRAKLTISPDYAYGATGHPGIIPPHATLV FDVELLKLEGS $(33,39)$.

\section{lys2::ins $E-A_{14}$ reversion assay (Tables 1,2 )}

Assays were performed as described previously (9). Briefly, strains listed in Supplementary Table S1 were freshly struck from frozen stocks and grown in synthetic complete media in the presence or absence of rapamycin $(2 \mu \mathrm{g} / \mathrm{ml})$ and then inoculated in liquid complete media maintaining either the presence or absence of rapamycin prior to plating onto complete and lysine dropout plates. Strains containing plasmids were grown in minimal selective leucine dropout, to maintain pEAA213, or uracil dropout, to maintain pRS416 and pEAA67. Rapamycin was included in growth media until just before cell cultures were plated onto complete and lysine dropout plates to measure lys $2::$ ins $E$ - $A_{14}$ reversion. Rates of lys $2:$ ins $E-A_{14}$ reversion were calculated as $\mu=$ $f / \ln (N \cdot \mu)$, where $f$ is reversion frequency and $N$ is the total number of revertants in the culture (42). For each strain, 15-64 independent cultures, obtained from two to three independent transformants bearing a unique allele, were assayed on at least two different days to prevent batch effects, and $95 \%$ confidence intervals and all computer-aided rate calculations were performed as described previously $(9,43)$.
For the post-rapamycin treatment described in Table 1, the wild-type and Complex \#1 strains were grown both in the presence and absence of rapamycin $(2 \mu \mathrm{g} / \mathrm{ml})$ and then inoculated in liquid complete media maintaining either the presence or absence of rapamycin prior to plating onto complete and lysine dropout plates. Upon colony counting after 3 days of incubation, a single colony from the complete plate was inoculated in liquid media lacking rapamycin and then assayed again for mutator phenotype.

\section{Biochemical assays}

Cross-linking mass spectrometry (XL-MS). Mlh1-Pms1 and Complex \#5 were crosslinked with disuccinimidyl sulfoxide (DSSO; Thermo Fisher Scientific). A $50 \mathrm{mM}$ stock solution of DSSO was freshly prepared by dissolving DSSO into anhydrous dimethyl sulfoxide (DMSO). $5 \mu \mathrm{g}$ of Mlh1Pms 1 and Complex \#2 and \#5 derivatives were incubated in $50 \mu \mathrm{l}$ of buffer containing $25 \mathrm{mM}$ HEPES $\mathrm{pH} 8.0,180 \mathrm{mM}$ $\mathrm{NaCl}, 10 \%$ glycerol). DSSO was included at a final concentration of $1.25 \mathrm{mM}$ and the reaction was then incubated for $30 \mathrm{~min}$ at room temperature. Reactions were quenched by the addition of Tris- $\mathrm{HCl} \mathrm{pH} 8.0$ to a final concentration of $10 \mathrm{mM}$. Samples were digested and processed for MS as described in Yugandhar et al. (44). In brief, the crosslinked samples were denatured with $1 \%$ sodium dodecyl sulfate (SDS) at $65^{\circ} \mathrm{C}$ for $15 \mathrm{~min}$, reduced by $1 \mathrm{mM}$ dithiothreitol (DTT) at room temperature for $15 \mathrm{~min}$, and then alkylated with $25 \mathrm{mM}$ iodoacetamide at room temperature for $15 \mathrm{~min}$. Proteins were precipitated by adding $3 \times$ volumes of cold acetone/ethanol/acetic acid solution (50:49.9:0.1, $\mathrm{v} / \mathrm{v} / \mathrm{v})$. The precipitates were resuspended in $8 \mathrm{M}$ urea, 50 $\mathrm{mM}$ Tris- $\mathrm{Cl}$ and $150 \mathrm{mM} \mathrm{NaCl}, \mathrm{pH}$ 8.0. After dilution to $2 \mathrm{M}$ Urea, trypsin (Trypsin Gold, Promega, Madison, WI) digestion was performed at $37^{\circ} \mathrm{C}$ overnight. Trifluoroacetic acid-formic acid (TFA-FA) solution was applied to terminate digestion. The digested peptides were desalted using Sep-Pak C18 cartridge (Waters, Dublin, Ireland), dried using SpeedVac ${ }^{\mathrm{TM}}$ Concentrator (Thermo Fisher Scientific, Pittsburgh, PA) and stored in $-80^{\circ} \mathrm{C}$ for further analysis.

Peptides were resuspended in $0.1 \%$ TFA and then analyzed using an Orbitrap Fusion Lumos Tribrid mass spectrometer (Thermo Fisher Scientific) coupled online to an EASY-nLC 1200 system (Thermo Fisher Scientific) equipped with a $75 \mu \mathrm{m} \times 25 \mathrm{~cm}$ capillary column (in-house packed with $3 \mu \mathrm{m} \mathrm{C18}$ resin; Michrom BioResources). For each analysis, peptides were eluted using a 45 min liquid chromatography gradient $(5 \% \mathrm{~B}-40 \% \mathrm{~B}$; mobile phase A composed of $0.1 \%$ formic acid (FA), and mobile phase B composed of $0.1 \%$ FA and $80 \%$ acetonitrile $(\mathrm{ACN})$ ) at a flow rate of $200 \mathrm{nl} / \mathrm{min}$.

The CID-MS2-HCD-MS3 acquisition method was used for DSSO-crosslinking identification. The MS1 precursors were detected in the Orbitrap mass analyzer at resolution of 60000 with a scan range from $375 \mathrm{~m} / z$ to $1500 \mathrm{~m} / \mathrm{z}$. The precursor ions with the charge of +4 to +8 were selected for MS2 analysis at a resolution of 30000 (AGC target $=1 \times 10^{5}$, precursor isolation width $=1.6 \mathrm{~m} / \mathrm{z}$, and maximum injection time $=10^{5} \mathrm{~ms}$ ), with the normalized collision energy of CID at $25 \%$. The two most abundant reporter ions with a mass difference of $31.9721 \mathrm{Da}$ in CID- 
MS2 spectra were selected for further MS3 analysis. The selected ions were fragmented in an Ion Trap using HCD with the normalized collision energy at $35 \%$ and AGC target of $2 \times 10^{4}$. All spectra were recorded by Xcalibur 4.1 software and Orbitrap Fusion Lumos Tune Application v. 3.0 (Thermo Fisher Scientific).

The cross-link search was performed using MaXLinker software (44). The wildtype and engineered Mlh1-Pms1 cross-linked samples were searched against database with corresponding target and randomized sequences, along with randomized sequences of $E$. coli proteome (5268 sequences) for efficient FDR estimation. Crosslink maps for Mlh1-Pms1 are a composite of results from two independent crosslink trials.

Protein purification. Mlh1-Pms1 and Complex \#1 to \#5 variants (Supplementary Figure S1B) were purified as described from galactose-induced cultures of BJ2168 (MATa, ura3-52, leu2-3,112, trp1-289, prb1-1122, prc1-407, pep4-3) bearing pEAE expression vectors (Supplementary Tables S1, S2; 23,41). The $M L H 1$ expression constructs pEAE 446-448 contain a FLAG tag at position 499 (with respect to the wild-type sequence). RFC and PCNA were expressed and purified from E. coli $(45,46)$. All biochemical assays were performed with at least two independently purified Mlh1-Pms1 complexes.

DNA substrates for biochemical assays. Closed circular pUC18, and when specified pUC19 (both are $2.7 \mathrm{~kb}$ and are nearly identical in sequence, with only the polylinker regions reversed, Thermo Fisher Scientific, Waltham, MA), were used as DNA substrates in the endonuclease assays presented in Figures 3 and 4, and Supplementary Figures S2 to S4. A 49-mer homoduplex DNA substrate was included in the ATPase experiments presented in Figures 3B, 4B and Supplementary Figure S2B. This substrate was made by annealing AO3142 (5'GGGTCAACGTGGGCAAAG ATGTCCTAGCAAGTCAGAATTCGGTAGCGTG) and AO3144 (5'CACGCTACCGAATTCTGACTTGCT AGGACATCTTTGCCCACGTTGACCC). AO3142 and $\mathrm{AO} 3144$ were added at an equal molar ratio in buffer containing $10 \mathrm{mM}$ Tris- $\mathrm{HCl}, \mathrm{pH} 7.5,100 \mathrm{mM} \mathrm{NaCl}, 10$ $\mathrm{mM} \mathrm{MgCl} 2$ and $0.1 \mathrm{mM}$ EDTA. These oligonucleotides were annealed through an incubation at $95^{\circ} \mathrm{C}$ for $5 \mathrm{~min}$, followed by cooling to $25^{\circ} \mathrm{C}$ at a rate of $1{ }^{\circ} \mathrm{C} / \mathrm{min}$. Following annealing, excess single-stranded DNA was removed using an S300 spin column (GE Healthcare). For the microscale thermophoresis (MST) analysis presented in Figures 3A, 4A, Supplementary Figure S2A, a 48-mer homoduplex DNA substrate was made by annealing AO4549 (5'-6-FAM-CTGGACGGGTTAAGACCGAA CGTGGCTCCAGAAACGGGTGCAACTGGG; synthesized by Integrated DNA technologies) and $\mathrm{AO} 4548$ (5'CCCAGTTGCACCCGTTTCTGGAGCCACGTTCG GTCTTAACCCGTCCAG) as described above.

Endonuclease assays. Reactions were performed in $20 \mu \mathrm{l}$ in $1 \times$ endonuclease buffer ( $20 \mathrm{mM}$ HEPES-KOH ( $\mathrm{pH} 7.5)$, $20 \mathrm{mM} \mathrm{KCl}, 2.5 \mathrm{mM} \mathrm{MnSO} 4,0.2 \mathrm{mg} / \mathrm{ml} \mathrm{BSA}, 1 \%$ glycerol; Ref. 47). Mlh1-Pms1, Complex \#1, Complex \#2, or Complex \#5 were included at final concentrations of 6.25
$100 \mathrm{nM}$. When indicated, rapamycin was included at a final concentration of $1 \mu \mathrm{M}$. Rapamycin was dissolved into DMSO at a concentration of $10 \mathrm{mM}$ then serially diluted in DMSO to a final concentration of $100 \mu \mathrm{M}$. The 100 $\mu \mathrm{M}$ solution was then diluted in $1 \times$ endonuclease reaction buffer to a concentration of $20 \mu \mathrm{M}$ before being added to individual reaction tubes at a final concentration of 1 $\mu \mathrm{M}$. Rapamycin was added to the reaction before the addition of the DNA substrate and the reaction was incubated at $37^{\circ} \mathrm{C}$ for $5 \mathrm{~min}$ prior to DNA addition. Reactions $\left(37^{\circ} \mathrm{C}, 40 \mathrm{~min}\right)$ were started following the addition of $5.1 \mathrm{nM}$ final concentration of pUC18 (or pUC19 when indicated, no differences were seen for MLH endonuclease activity on these substrates) and stopped by the addition (final concentrations shown) of $0.1 \% \mathrm{SDS}, 14 \mathrm{mM}$ EDTA and $0.1 \mathrm{mg} / \mathrm{ml}$ Proteinase K (New England Biolabs). DNA was electrophoresed in $1.2 \%$ agarose gels in 1xTAE (Tris-acetate-EDTA) containing $0.1 \mathrm{gg} \mathrm{m}^{-1}$ ethidium bromide, which causes covalently closed circular DNA isoforms to separate from nicked DNA product. Gels were run in $1 \times \mathrm{TAE}$ at $100 \mathrm{~V}$ for $45 \mathrm{~min}$. Negative control lanes were used as background and were subtracted out of reported quantifications. BioRad Image Lab Software, v5.2.1 was used to quantify gels.

ATPase assays. ATPase activity was determined using the Norit A absorption method described previously $(9,47)$. Briefly, $30 \mu \mathrm{l}$ reactions contained $0.4 \mu \mathrm{M}$ of Mlh1-Pms1, Complex \#2, or Complex \#5, $100 \mu \mathrm{M}$ [ $\gamma$-32P]-ATP, $20 \mathrm{mM}$ Tris, pH 7.5, $2.0 \mathrm{mM} \mathrm{MgCl}_{2}, 0.1 \mathrm{mM}$ DTT, $1 \mathrm{mM} \mathrm{MnSO}_{4}$, $75 \mathrm{mM} \mathrm{NaCl}, 1 \%$ glycerol, $40 \mu \mathrm{g} / \mathrm{ml}$ BSA. Reactions were incubated for $40 \mathrm{~min}$ at $37^{\circ} \mathrm{C}$. When specified, DNA (49mer homoduplex DNA substrate), PCNA, and rapamycin were included at $0.75,0.5$ and $1 \mu \mathrm{M}$, respectively. Just prior to addition, rapamycin was diluted and included in reactions as described for the endonuclease assays.

Microscale thermophoresis (MST) assay. MST was performed using a Monolith NT.115 instrument (NanoTemper Technologies) equipped with red and blue filters using methods described in 48). MLH complexes were serially diluted 16 times in 2-fold steps to final concentrations of 4400 to $2.14 \mathrm{nM}$ for Mlh1-Pms1, 4000 to $1.95 \mathrm{nM}$ for Complex \#2, and 4800 to $2.34 \mathrm{nM}$ for Complex \#5, and then mixed with an equal volume of a $40 \mathrm{nM}$ solution of 6-FAM-labeled duplex oligonucleotide (sequence described above). Reaction buffer contained $20 \mathrm{mM}$ Tris- $\mathrm{HCl}(\mathrm{pH}$ 7.5), $20 \mathrm{mM} \mathrm{NaCl}, 0.01 \mathrm{mM}$ EDTA, $2 \mathrm{mM} \mathrm{MgCl} 2,40$ $\mu \mathrm{g} / \mathrm{ml}$ BSA, $0.1 \mathrm{mM}$ DTT and $0.05 \%$ TWEEN-20. Assays with nucleotide, rapamycin, or both contained $1 \mathrm{mM}$ ATP and $1 \mu \mathrm{M}$ rapamycin. ATP and rapamycin were added to reactions containing Mlh1-Pms1 prior to DNA addition. The reactions ( $20 \mu \mathrm{l}$ volumes) were then incubated for $5 \mathrm{~min}$ at room temperature. Following DNA addition, reactions were incubated at room temperature at $30^{\circ} \mathrm{C}$ for $15 \mathrm{~min}$. They were then loaded into standard capillaries (NanoTemper Technologies). Reactions were then tested with $40 \%$ excitation power, medium MST power, and measured using M.O. Control software (NanoTemper Technologies). M.O. Affinity Analysis software (version 2.3, Nanotemper Technologies) was used to analyze data and determine the nor- 
malized fluorescence $\left(F_{\text {nom }}\right)$ for each concentration. $F_{\text {norm }}$ is calculated by dividing $F_{\text {hot }}$ (average fluorescence value in the heated state) by $F_{\text {cold }}$ (average fluorescence value measured in the cold state before the infrared (IR) laser is turned on) and plotted as parts per thousand (\%). Three independent reactions were measured to obtain $F_{\text {norm }}$ values, which were then averaged (mean \pm standard deviation) and plotted against the respective concentration of Mlh1-Pms1 (Figures 3A, 4A, Supplementary Figure S2A). Binding constants $\left(K_{\mathrm{d}}\right)$ were determined by nonlinear curve fitting using GraphPad Prism 9. All experiments were performed using at least two independently purified proteins.

\section{RESULTS}

Mlh1-Pms1 inter-subunit interactions involve the $\mathrm{N}$ terminal, IDR and C-terminal domains of both proteins

We used cleavable cross-linking coupled with mass spectrometry (XL-MS) as a tool to map intra- and inter-subunit interactions in Mlh1-Pms1. In this approach, disuccinimidyl sulfoxide (DSSO; Materials and Methods) was used to cross-link exposed lysine residues in close proximity (10.1 angstrom spacer length; 44,49). While this method cannot yield detailed structural information, it can identify regions of proteins that potentially interact and thus give insights into the movements of Mlh1-Pms1 during MMR. We were unable to perform XL-MS in the presence of ATP or the non-hydrolysable ATP analog AMP-PMP because the primary amines present in these molecules interfered with our detection of DSSO-induced cross-linking by mass spectrometry. Also, we did not attempt XL-MS with an ATP analog that lacks the primary amine, 6-methyl ATP, because this analog did not activate Mlh1-Pms1's latent endonuclease activity.

We identified large numbers of crosslinks involving the IDRs in Mlh1-Pms1 (76 of 102 crosslinks; Figure 2A and Supplementary Table S3), indicating that the IDRs are highly flexible, solvent exposed, and are capable of interacting with nearby partners. Crosslinks were also seen between the Mlh1 and Pms1 C-terminal domains, consistent with the C-terminal domains being required for Mlh1-Pms1 interactions (32), and between the ATP binding (N-terminal) domains of Mlh1 and Pms1, consistent with structural and biochemical studies showing that these domains interact when both domains are ATP bound $(11,30)$. Interestingly, many of the 15 inter-subunit crosslinks map to regions of Mlh1 and Pms1 that are genetically critical for their function (see asterisks in Figure 2A, Supplementary Table S4; 11,23,25,50). Lastly, crosslinks were identified between the Mlh1 IDR and the Pms1 C-terminal endonuclease domain, providing evidence for models in which the condensation of the $\mathrm{N}$ - and C-termini of MLH proteins is linked to the activation of MLH functions (21,30-32).

\section{Rapamycin-induced disruption of MMR in vivo}

The identification of regions within the IDRs of Mlh1 and Pms1 that had few if any crosslinks (Figure 2A) and could be deleted without impacting MMR functions (Supplementary Figure S1A; 23) encouraged us to develop a method to restrict any potential coordinated interactions that drive
MMR functions. We employed a chemically inducible system to control the dimerization of a pair of proteins with a small molecule that acts as a 'dimerizer'. Dimerizers can reversibly bring regions of proteins together that normally lack interaction and have been used to study mechanistic aspects of protein localization, sister chromatid cohesion, targeted protein degradation, transcriptional control and signal transduction $(36,38,39,51-53)$. Specifically, we targeted the Mlh1 and Pms1 IDRs through the $11 \mathrm{kDa}$ FKBPrapamycin binding (FRB) domain of the mammalian target of rapamycin protein (mTOR) protein and the $12-\mathrm{kDa}$ FK506 binding protein (FKBP; Figure 2A; 33). The FRB (93 amino acids) and FKBP (107 amino acids) polypeptides are relatively small and each bind to the small molecule macrolide rapamycin. Rapamycin binding to each domain facilitates a stable and tight FRB-FKBP heterodimer $\left(K_{\mathrm{d}}\right.$ in the sub $\mathrm{nM}$ range; 54,55). We first tested if we could reversibly inhibit MMR by restricting the motion of the linker arm of Mlh1 in the presence of rapamycin (Complex \#1, Figure 2B). We chose Mlh1 as our initial target because Mlh1 has been proposed to initiate Mlh1-Pms1 IDR condensation (Figure 1B; 4,21,27). We then tested the effects of disrupting the Mlh1 IDR movement genetically, using a reversion assay with a wide range of sensitivity, and biochemically, by measuring Mlh1-Pms1 DNA binding, ATPase, and endonuclease activities.

In the first experiment, FRB and FKBP were introduced in tandem into the Mlh1 IDR (Figure 2B, Supplementary Figure S1A). The effects of these insertions were assessed using a highly sensitive reversion assay in which an insertion of 14 adenosine residues disrupts the LYS2 open reading frame $\left(\right.$ lys $\left.2-A_{14}\right)$. Frameshift mutations (primarily -1 ) which restore the open reading frame are detected as Lys ${ }^{+}$ colonies. An $m l h l \Delta$ strain shows a nearly four-orders of magnitude (5000 to 6000 -fold) higher rate of reversion to Lys $^{+}$compared to wild-type $(9,42)$. As shown in Table 1, single insertions of FRB or FKBP in Mlh1 conferred reversion rates that were only marginally higher than wild-type (1.8- to 4.9-fold). As shown in Table 1, the double insertion allele, $m l h 1-F R B_{355}-F K B P_{464}$, (referred to as Complex \#1) conferred a very weak reversion phenotype (51.9-fold). The single domain insertion $m l h l$ alleles conferred higher reversion rates when rapamycin was added (68- to 114 -fold), but to a much lower level than seen for $m l h 1 \Delta$ (5400-fold). One reason for the modest increase in reversion rate for the single insertion strain grown with rapamycin is that both FRB and FKBP bind rapamycin, and such binding could impact MMR by altering Mlh1 IDR flexibility. Alternatively, rapamycin binding proteins in the yeast cell other than Fpr1 (the yeast homolog of human FKBP, deleted in our strain background) may be able to weakly interact with FRB or FKBP, blocking Mlh1 from interacting with partners or mismatch substrates. Consistent with the latter idea is that the single domain insertion alleles were recessive (Table 2); if rapamycin impacted IDR function, one might have expected to still see a mutator phenotype when the FRB and FKBP insertion alleles were expressed in the presence of wild-type $M L H 1$, though at lower levels (see below).

In contrast to the single $F R B$ and FKBP mlhl insertion strains grown in the presence of rapamycin, a complete MMR defective phenotype was observed for the $m l h 1$ - 


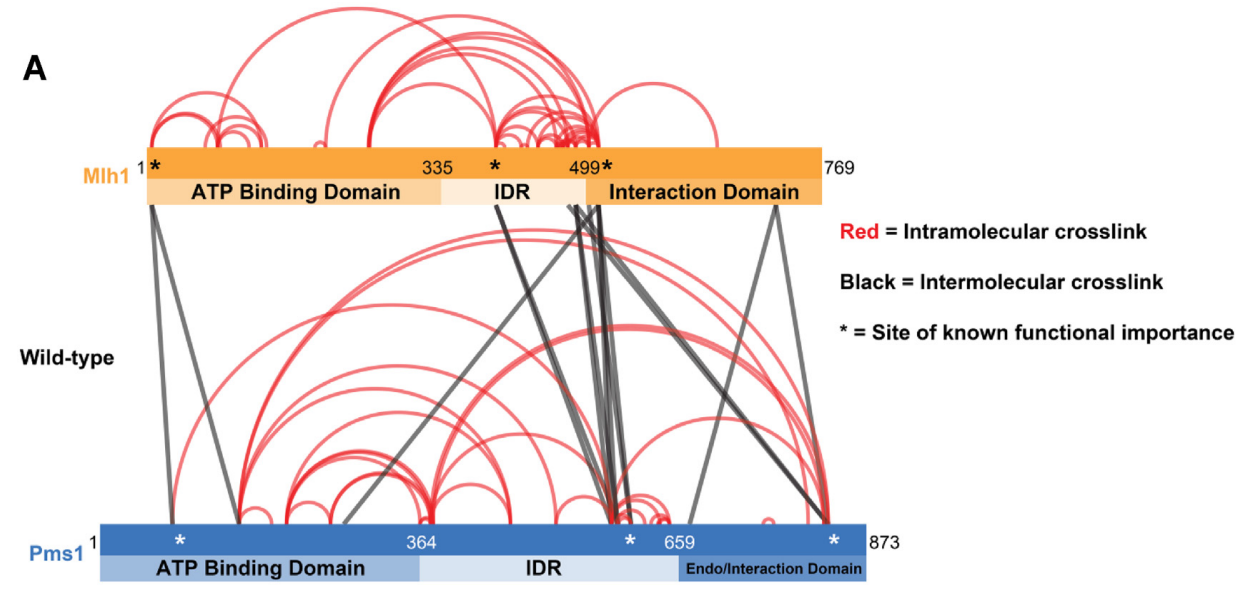

B
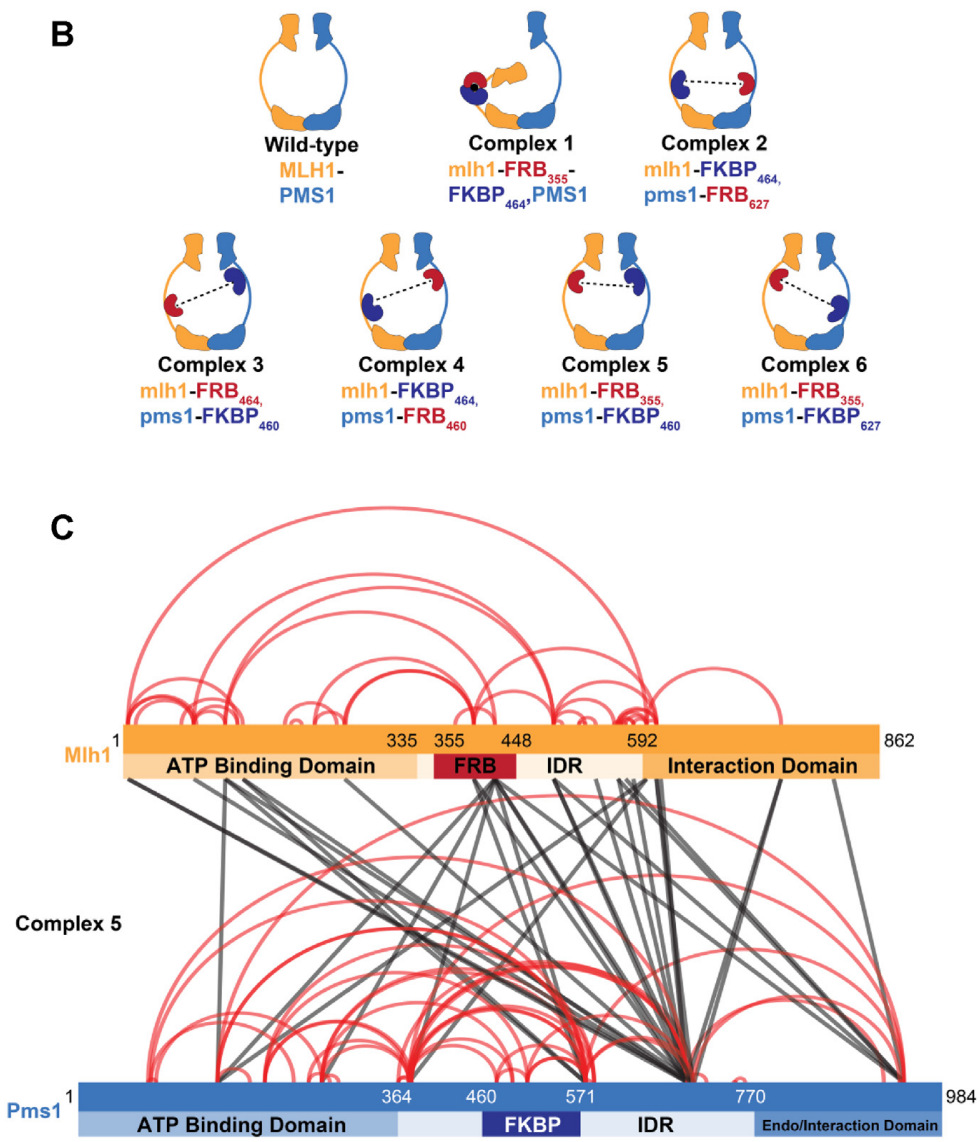

Figure 2. Crosslinking mass spectrometry of Mlh1-Pms1 identifies IDR regions that display limited or extensive interactions within a subunit or with the other subunits. (A) Mlh1-Pms1 was crosslinked with DSSO and then subjected to cross-linking mass spectrometry (XL-MS; Materials and Methods; Supplementary Table S3). The positions for each domain are shown. For Mlh1, the ATP binding domain extends from aa position 1-335; the IDR from 336-498; and the interaction domain from 499-769. For Pms1 the ATP binding domain extends from aa position 1-364; the IDR from 365-659; and the endonuclease/interaction domain from 660-873. Shown are the inter- and intra-subunit lysine residue crosslinks with respect to the ATP binding, IDR, and endonuclease/interaction domains of Mlh1 and Pms1. Crosslinks involving the same positions (indicated by the density of the line) were seen multiple times in a single experiment and were also identified in a repeat experiment. For simplicity, we show the composite of the results from two independent crosslink trials. * Represent positions in Mlh1 and Pms1 shown previously to disrupt MMR. (B) Cartoons of Complexes 1 to 6 analyzed in this study. Crosslinking analysis and previous deletion analysis of Mlh1 and Pms1 IDRs (23; Supplementary Figure S1A) encouraged is to insert FRB and FKBP domains into the IDRs of Mlh1 and Pms1 at amino acid positions 355 or 464 in Mlh1 or 460 or 627 in Pms1, with the specific insertion sites of the FRB and FKBP domains in Complexes 1 to 6 indicated. Potential interactions with rapamycin are indicated by the dotted black lines. (C) XL-MS analysis of Complex \#5 (mlhl-FRB 355 , pmsl-FKBP 460$)$ with the insertions of FRB and FKBP indicated. The positions of each domain are shown but are shifted by 97 amino acids in Mlh1 with the insertion of FRB (93 amino acids plus four spacer amino acids), and 111 amino acids in Pms1 for the insertion of FKBP (107 amino acids plus four spacer amino acids). 
Table 1. FRB and FKBP domain insertions into the MLHI and PMSI IDRs confer MMR defects in the presence of rapamycin

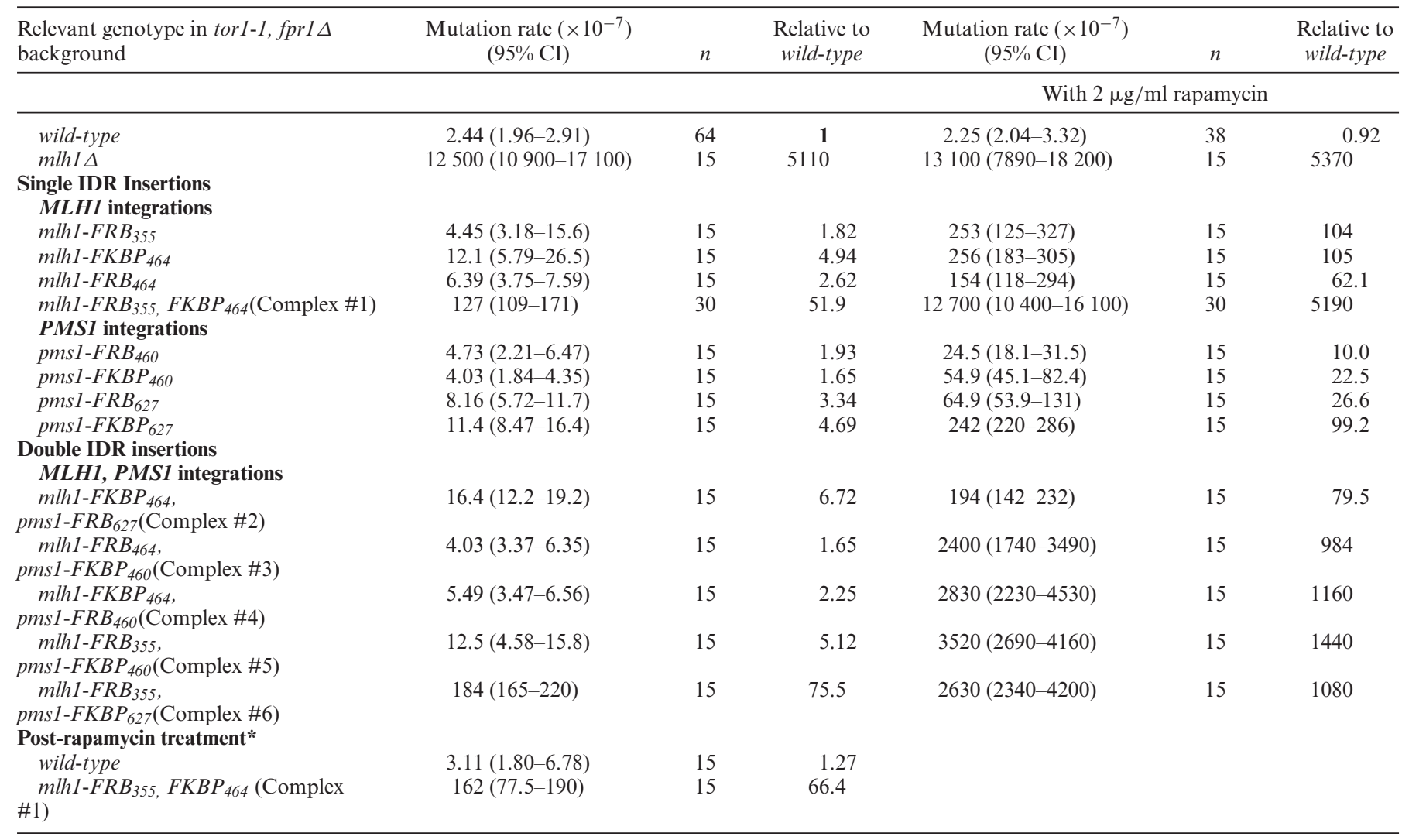

The indicated alleles were integrated into their native $M L H 1$ or $P M S 1$ loci in the S288C strain background. Strains were tested for DNA mismatch repair functions in the presence and absence of $2 \mu \mathrm{g} / \mathrm{ml}$ of rapamycin using the $l y s 2-A_{14}$ reversion assay (Materials and Methods) with $95 \%$ confidence interval (CI) presented. The wild-type strain for these experiments, EAY4450, was generated by deleting the FPR1 locus and introducing the torl-1 (S1972A) allele into EAY1269. n represents the number of independent measurements from at least two transformants. ${ }^{*}$ Wild-type and $m l h 1-F R B_{355}$, $F K B P_{464}-$ PMS1 (Complex \#1) strains were grown in the presence of rapamycin, after which independent colonies from these strains were grown in the absence of rapamycin, and then the mutation rate was determined.

$F R B_{355}-F K B P_{464}$ double insertion allele (Complex \#1) in the presence of rapamycin, a phenotype identical to that seen when the Mlh1 IDR was completely deleted (23). We then asked if MMR can be re-initiated when rapamycin is washed out of the media in mlh1-FRB $B_{355}-F K B P_{464}$ strains. With rapamycin removed, MMR activity of the mlhl$F R B_{355}-F K B P_{464}$ (Complex \#1) strain returned to prerapamycin levels (Table 1). This indicates that MMR functions can be reversibly and specifically modulated using a small molecule.

\section{Rapamycin-induced interactions between Mlh1 and Pms1 IDRs disrupt MMR}

MLH clamp formation is hypothesized to create a condensed state poised for endonuclease activation upon encountering PCNA on DNA $(9,13,27)$. Based on this model we thought that restricting MLH clamp formation through FRB-FKBP interactions would inhibit MMR, and that such inhibition could be detected in biochemical assays. We constructed five additional Mlh1-Pms1 FRB/FKBP insertion complexes that maintained roughly wild-type levels of MMR in the absence of rapamycin (Figure 2B). This wildtype function was also seen when one subunit contained the FRB/FKBP insertion and the other a wild-type partner, in- dicating that the FRB/FKBP insertions were well tolerated (Table 2).

Complexes that restrain the IDRs of Mlh1 or Pms1 near the N-terminal region displayed similar and strong MMR defects in the presence of rapamycin (Complexes 3-6; 984to 1440 -fold higher reversion rate to Lys ${ }^{+}$; Table 1). Complex \#2, predicted to restrain the IDRs of Mlh1 and Pms1 near their C-terminal regions, conferred weak MMR defects (79.5-fold). Interestingly, the small increase in reversion rate for Complex \#2 seen in the presence of rapamycin was very similar to the rate seen when the FRB and FKBP insertion alleles in Complex \#2 were expressed in the presence of a wild-type partner, indicating that the effect of rapamycin on Complex \#2 function was minimal (Table 1). One explanation for these phenotypes is that interactions predicted to restrain the coordinated movements of the Nterminal ATP binding domains (Complexes 3-6) disrupt MMR whereas those predicted to maintain free movement of the N-terminal ATP binding domains (Complex \#2) do not. However, we cannot exclude the possibility that the efficiency of FRB-FKBP dimerization is compromised for Complex \#2.

We asked if Complexes 2-6 expressed in the presence of similar levels of wild-type complex would display evidence of subunit mixing. If this was the case, then one quar- 
Table 2. Analysis of FRB and FKBP insertions in the MLH1 and PMSI IDRs in strains also bearing wild-type copies of $M L H 1$ and PMS1

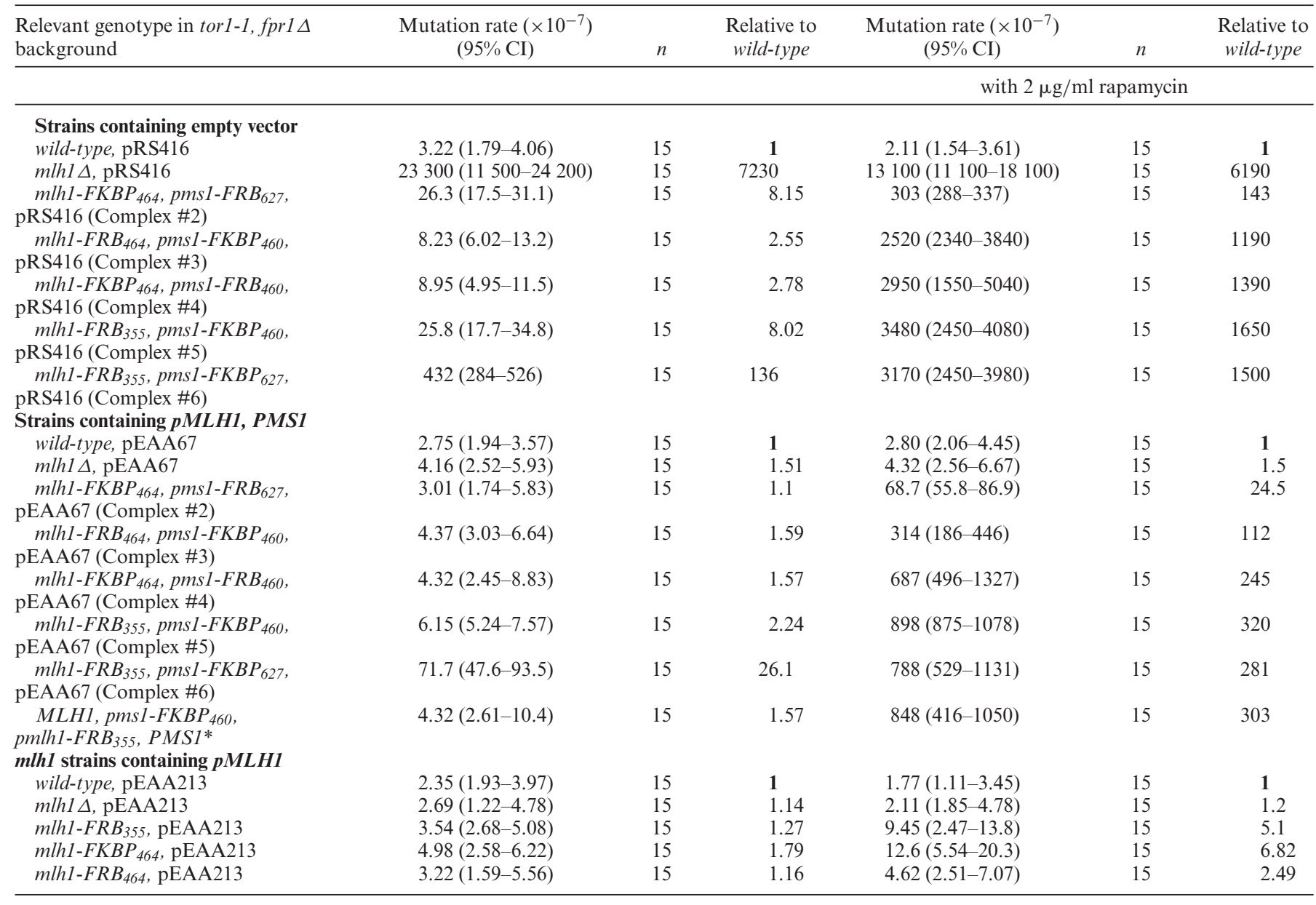

The indicated strains were transformed with either an empty vector (pRS416), an ARS-CEN plasmid expressing $M L H 1, P M S 1, M S H 2$ (pEAA67), or an $A R S-C E N$ plasmid expressing $M L H 1$ (pEAA213). Strains were tested for DNA mismatch repair functions both in the presence and absence of $2 \mu \mathrm{g} / \mathrm{ml}$ of rapamycin using the $l y s 2-A_{14}$ reversion assay (Materials and Methods). * EAY4495 (relevant genotype $M L H 1$, pms1-FKBP 460) was transformed with an $A R S$ - $C E N$ plasmid expressing $m l h 1$-FRB355 (pEAA672) and an ARS-CEN plasmid expressing PMSI (pEAA248). 95\% confidence intervals (CI) are presented. $\mathrm{n}$ represents the number of independent measurements from at least two transformants.

ter of the complexes in a cell would be expected to contain FRB and FKBP insertions in the same heterodimer (evidence for intra-complex interactions), and the reversion rate for cells expressing both the complex and wildtype Mlh1-Pms1 in the presence of rapamycin would be expected to be one quarter of the rate seen when only the complex was expressed. If inter-complex interactions were prevalent then we would have expected to see at most a twofold reduction in reversion rate. As shown in Table 2, the reversion rates for the five complexes expressed in the presence of rapamycin and wild-type Mlh1-Pms1 were roughly one quarter of the rates seen when only the complexes containing FRB and FKBP domains were expressed. Specifically, Mlh1-Pms1 presence/absence reversion rate ratios were $0.23,0.12,0.23,0.26$ and 0.25 for Complexes $2,3,4,5$ and 6 , respectively. These observations are consistent with rapamycin inducing interactions between FRB and FKBP insertions within single heterodimers. They are also consistent with the determination that the local concentrations of the FRB and FKBP domains within a single heterodimer are expected to be much higher (estimated to be as high as three to four orders of magnitude) than between separate Mlh1-Pms1 molecules, which are present at $\sim 600$ copies in a single yeast nucleus (56). This calculation is based on a haploid yeast nucleus volume of $2.9 \pm 0.9 \mu \mathrm{m}^{3}$ (57) and Mlh1-Pms1 molecules in a haploid yeast nucleus diffusing randomly throughout the nuclear volume, with the motion of FRB and FKBP within the IDRs of the Mlh1-Pms1 heterodimer restricted to a shell of an approximately $10 \mathrm{~nm}$ radius (19).

The experiments presented in Table 2 and our calculation of Mlh1-Pms1 concentrations in vivo encouraged us to purify Complexes 1-5. These complexes formed stable heterodimers, remained soluble when rapamycin was added in solution, and for those tested, retained ATPase and endonuclease activities (Supplementary Figure S1B and below). Because Mlh1-Pms1 forms oligomers on DNA (22), we were concerned that the addition of rapamycin could cause neighbouring Mlh1-Pms1 complexes to aggregate and become insoluble. However, these proteins retained solubility 
and activity in the presence of rapamycin, providing further evidence for rapamycin promoting intra-complex interactions.

\section{Rapamycin induced N-terminal dimerization prevents Com- plex \#5 from stably binding to DNA}

MLH proteins display DNA binding activities that are required for their MMR functions $(20,23)$. To test if restriction of the IDRs of Mlh1-Pms1 affected DNA binding, we assayed the biochemical activities of Mlh1-Pms1, Complex \#1, Complex \#2 and Complex \#5 in DNA binding, ATPase, and endonuclease assays. These complexes were chosen because strains bearing the corresponding mutant alleles showed a representative range of MMR defects in the absence and presence of rapamycin. Motivated by the XLMS results, we first present biochemical analyses of Complexes 2 and 5 in which we restricted interactions between the Mlh1 and Pms1 IDRs. We then present the biochemical analysis of Complex \#1 in which only the Mlh1 IDR was restricted.

We examined the binding of Complex \#5, and Complex \#2) to a 49 bp homoduplex oligonucleotide using Microscale Thermophoresis (MST). As shown in Figure 3A and Table 3, wild-type Mlh1-Pms1 affinity for DNA was reduced in the presence of ATP (141 nM in its absence to $675 \mathrm{nM}$ in its presence). This binding was unaffected by the presence of rapamycin. The reduced binding of Mlh1-Pms1 in the presence of ATP is consistent with previous studies indicating that Mlh1-Pms1 ring opening and closing is linked to interactions with DNA $(9,19,20)$. In the absence of rapamycin, Complex \#5 DNA binding affinities were similar to Mlh1-Pms1 in both the presence and absence of ATP. The addition of rapamycin significantly reduced the DNA binding affinity for Complex \#5 in the absence of ATP (775 $\mathrm{nM}$ for Complex \#5 versus $173 \mathrm{nM}$ for Mlh1-Pms1). The $K_{\mathrm{d}}$ for binding of Complex \#5 in the presence of ATP and rapamycin was too weak to be determined due to the aggregation of Mlh1-Pms1 at high concentrations; however, the lower limit for the $\mathrm{K}_{\mathrm{d}}$, based on $\sim 50 \%$ binding, was estimated to be $5 \mu \mathrm{M}$ compared to $628 \mathrm{nM}$ for Mlh1-Pms1. In contrast, Complex \#2, which showed a mild mutator phenotype in the presence of rapamycin, displayed a DNA binding activity similar to wild-type both in the presence and absence of rapamycin (Supplementary Figure S2A; Table 3). Together these experiments argue that the restriction of the IDRs in Complex \#5 blocks the ability of Mlh1-Pms1 to form a stable complex on DNA, thus accounting for the MMR defect seen in vivo. It also matches with our previous observation that a deletion of the Pms1 IDR both disrupted DNA binding and prevented ternary complex formation of MSH and MLH proteins on a mismatched substrate (23).

\section{N-terminal dimerization in Complex \#5 pre-activates Mlh1- Pms1 in vitro}

We then assayed the activity of Complex \#2 and \#5 in ATPase and endonuclease assays (Figure 3B, Supplementary Figure S2B; 7,9,10). Previous studies showed that Mlh1Pms1 ATPase activity is stimulated in the presence of DNA duplex oligonucleotide and PCNA $(9,11-13,58)$. As shown in Figure 3B and Supplementary Figure S2B, we found that Mlh1-Pms1, Complex \#2, and Complex \#5 displayed, in the absence of rapamycin, similar ATPase activities. These activities were similar in both the presence or absence of DNA and PCNA. For Complex \#2, rapamycin did not affect ATPase activity in the presence or absence of DNA and PCNA. However, for Complex \#5 the addition of rapamycin resulted in significant increases in ATPase activities ( $\sim$ two-fold) both in the absence and presence of DNA and PCNA (Figure 3B).

We next measured the endonuclease activity of Complex \#2 and \#5 (Figure 3C, D; Supplementary Figure S2C, D). In the absence of rapamycin both complexes displayed comparable levels of PCNA stimulation of endonuclease activity compared to Mlh1-Pms1. We then performed these experiments in the presence of rapamycin and found that rapamycin did not impact Mlh1-Pms1 or Complex \#2 endonuclease activity; however, Complex \#5 displayed an endonuclease activity that was higher $(\sim 33 \%$ elevated) than seen for Mlh1-Pms1 (Figure 3D, Supplementary Figure S2D). Taken together, these observations indicate that Complex \#5 is inappropriately activated in a manner that disrupts MMR.

Finally, we analyzed Complex \#5 by XL-MS in the absence of rapamycin (Figure 2C, Supplementary Table S3). A similar number of crosslinks were observed for Complex \#5 (132, of which 94 involved IDRs) and Mlh1-Pms1 (102 crosslinks). 34 inter-subunit crosslinks were identified for Complex \#5, of which 25 were between Mlh1 and Pms1 residues. The remaining nine were between Pms1 and FRB residues. Of the 25 inter-subunit crosslinks involving Mlh1 and Pms1 in Complex \#5, 23 contained at least one residue that was involved in an inter-subunit crosslink in Mlh1Pms1. Lastly, 6 of the 15 inter-subunit crosslinks seen in Mlh1-Pms1 were also seen in Complex \#5. These observations provided evidence for conservation of inter-molecular interactions between Mlh1-Pms1 and Complex \#5. Our analysis of Mlh1-Pms1 (Figure 2A) indicated that the insertions of FRB and FKBP in Complex \#5 were in locations of the two proteins that were devoid of crosslinks, supporting the deletion analysis that these locations appear to not be critical for Mlh1-Pms1 function. We did not see any crosslinks in Complex \#5 between FRB and FKBP, consistent with genetic data indicating that these insertions did not interact unless rapamycin was present. We were unable to perform XL-MS in the presence of rapamycin because its addition interfered with sample preparation.

Seven novel cross-links, with some identified multiple times, were observed for Complex \#5 between the Mlh1 $\mathrm{N}$-terminal domain and the Pms1 IDR, reminiscent of the formation of the single-arm condensed complex involving the N-terminal domain of Mlh1 observed by Sacho et al. (21). We also saw a shift to form crosslinks between the Nterminal and IDR domains of Mlh1 and the IDR domain of Pms1. One explanation for these changes is that the addition of new FRB/FKBP domains into the IDRs is responsible for the novel crosslinks. Alternatively, the insertions shift Complex \#5 into a position where it is poised to perform the condensation steps that are normally seen only in the presence of ATP. Restricting such steps, as was done in the presence of rapamycin, could thus provide an explana- 
A

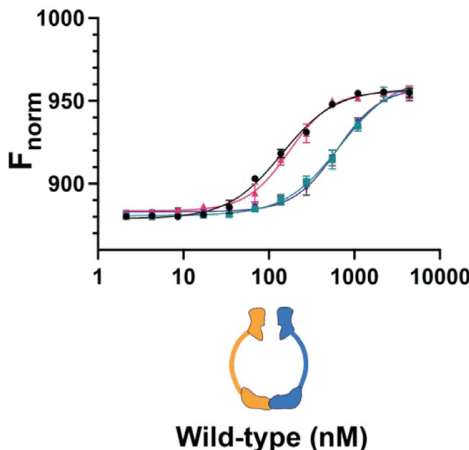

B with rapamycin

with duplex DNA, PCNA

with rapamycin, duplex DNA, PCNA

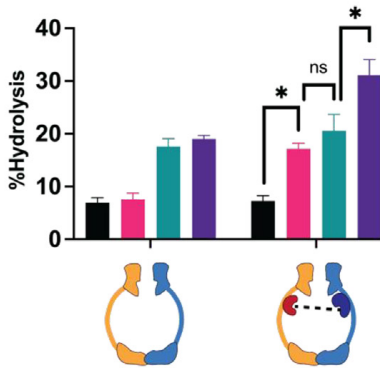

Wild-type Complex 5

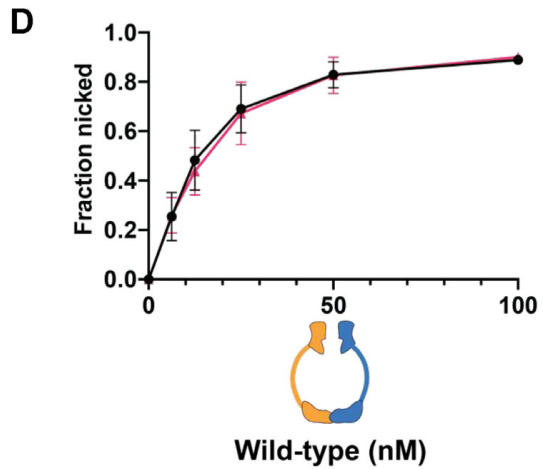

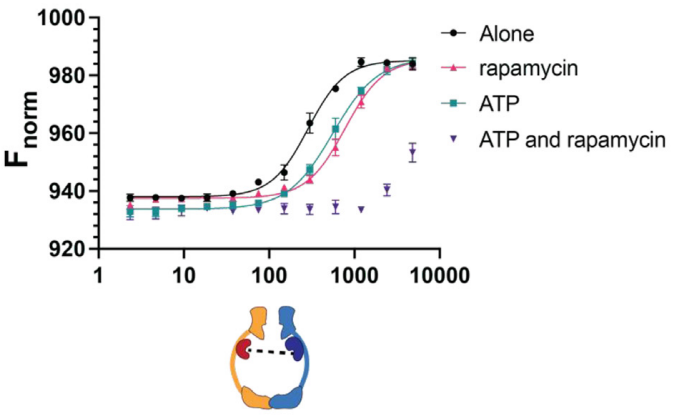

Complex $5(n M)$

C
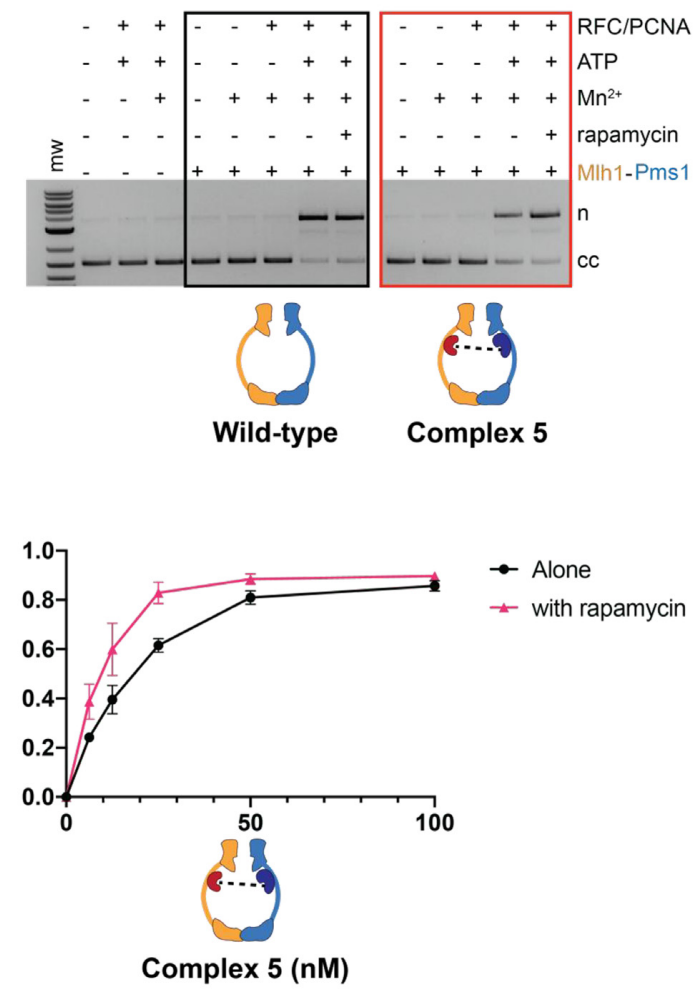

Figure 3. Complex \#5 (mlh1-FRB 355, pms1-FKBP 460$)$ displays defective DNA binding but enhanced ATP hydrolysis and endonuclease activity in the presence of rapamycin. (A) MST analysis of Mlh1-Pms1 and Complex \#5 in the presence and absence of 49 bp homoduplex DNA (20 nM), ATP (1 $\mathrm{mM}$ ) and rapamycin $(1 \mu \mathrm{M})$. Three independent experiments (error bars indicate the mean \pm standard deviation) and were performed using at least two independently purified batches of each protein. $\mathrm{F}_{\text {norm }}$ was calculated by dividing $\mathrm{F}_{\text {hot }}$ (average fluorescence value in the heated state) by $F_{\text {cold }}$ (average fluorescence value measured in the cold state before the infrared laser is turned on) and plotted as parts per thousand (\%). See Materials and Methods for details. (B) ATP hydrolysis activities of Mlh1-Pms1 and Complex \#5 (0.40 $\mu \mathrm{M}$ each) were determined in the presence and absence of PCNA (0.250 $\mu \mathrm{M})$, 49-bp homoduplex DNA $(0.75 \mu \mathrm{M})$, and rapamycin $(1 \mu \mathrm{M})$. Error bars indicate \pm one standard deviation for three replicates. * denotes statistical significance in a Student's $t$-test between the indicated comparisons; ns indicates not significant. (C) Endonuclease activities of Mlh1-Pms1 and Complex \#5 (50 nM each) determined on a closed circular DNA substrate (cc) in the presence $(+)$ or absence $(-)$ of $\mathrm{MnSO}_{4}, \mathrm{ATP}$, rapamycin, and yeast PCNA/RFC (Materials and Methods). $\mathrm{MnSO}_{4}$, ATP, rapamycin, RFC and PCNA were included at $5 \mathrm{mM}, 0.5 \mathrm{mM}, 1 \mu \mathrm{M}, 125 \mathrm{nM}$ and $250 \mathrm{nM}$, respectively. $\mathrm{n}=$ nicked product. (D) Endonuclease activities were determined at the indicated concentrations of wild-type and Complex \#5. Assays were performed in the presence of $\mathrm{MnSO}_{4}$, ATP, RFC, PCNA. Rapamycin was included as indicated. Error-bars indicate the standard deviation of three replicates (Supplementary Figure S3). 
Table 3. $K_{\mathrm{d}}$ measurements (nM) for MST DNA binding analysis

\begin{tabular}{lcccc}
\hline & alone & with ATP & with rapamycin & with rapamycin and ATP \\
\hline Wild-type & $141(128-157)$ & $675(584-803)$ & $173(157-192)$ & $628(563-709)$ \\
Complex \#1 & $98.4(88.9-110)$ & $421(378-526)$ & $198(167-220)$ & $1030(918-1240)$ \\
Complex \#2 & $207(182-235)$ & $431(380-494)$ & $211(186-238)$ & $391(347-444)$ \\
Complex \#5 & $287(264-312)$ & $556(510-610)$ & $775(709-855)$ & ND \\
\hline
\end{tabular}

$K_{\mathrm{d}}$ values, with $95 \%$ confidence intervals in parentheses, were determined in GraphPad Prism 9 through a nonlinear fit of graphs shown in Figures $3 \mathrm{~A}$, 4A and Supplementary Figure S2A. Curves were fit to data points representing the average of three independent assays. ND = not determined, due to incomplete saturation within the data acquisition range.

tion for the reduced MMR functions and inappropriate endonuclease activation seen for strains containing Complex \#5 grown in the presence of rapamycin.

\section{Rapamycin induced dimerization of FRB and FKBP within the MLH1 IDR in Complex \#1 disrupts Mlh1-Pms1 endonu- clease activity}

Kunkel and Erie (4) proposed that ATP binding to the Mlh1 subunit promotes the formation of an Mlh1-mediated onearmed collapsed complex, after which ATP binding to Pms1 yields a condensed activated state (Figure 1B). The finding that restricting the Mlh1 IDR with rapamycin conferred a null MMR phenotype ( $m l h 1-F R B_{355}, F K B P_{464}$ allele, Table $1)$ encouraged us to analyze the activity of Complex $\# 1$, which contains both the FRB and FKBP dimerization domains within the Mlh1 IDR. As shown in Figure 4A and Table 3, Complex \#1 displayed a modestly stronger DNA binding affinity $\left(K_{\mathrm{d}}=98 \mathrm{nM}\right.$ in the absence of ATP, 421 $\mathrm{nM}$ in the presence) compared to Mlh1-Pms1 ( $K_{\mathrm{d}}=141$ $\mathrm{nM}$ in the absence of ATP, $675 \mathrm{nM}$ in the presence). However, Complex \#1 and Mlh1-Pms1 showed similar $K_{\mathrm{d}}$ values in the presence of rapamycin (198 $\mathrm{nM}$ for Complex \#1, $173 \mathrm{nM}$ for Mlh1-Pms1); thus, rapamycin conferred a modest decrease in Complex \#1's DNA binding affinity ( $98 \mathrm{nM}$ in the absence to $198 \mathrm{nM}$ in the presence of rapamycin). Importantly, the addition of both ATP and rapamycin significantly reduced Complex \#1's DNA binding affinity $\left(K_{\mathrm{d}}\right.$ of $\left.1029 \mathrm{nM}\right)$, suggesting that restricting the Mlh1 IDR in Complex \#1 blocked its ability to form a stable ATP-dependent condensed complex on DNA.

To better understand why Complex \#1 displayed a nulllike MMR defect in the presence of rapamycin, we assayed the activity of Complex \#1 in ATPase and endonuclease assays (Figure 4B). The ATPase activities of wild-type and Complex \#1 were similarly induced by the presence of DNA and PCNA, and the presence of rapamycin did not affect this induction. However, the endonuclease activity of Complex \#1 was strongly affected by the addition of rapamycin. As shown in Figure 4C and Supplementary Figure S4A, in the absence of rapamycin, Complex \#1 (31.0 \pm 1.7 and $63 \pm 10 \%$ nicking at 25 and $50 \mathrm{nM}$ concentrations, respectively) displayed a somewhat weaker level of PCNA stimulation of endonuclease activity compared to Mlh1-Pms1 $(42.0 \pm 0.5$ and $75 \pm 12 \%$ nicking at 25 and $50 \mathrm{nM}$ concentrations, respectively). However, in the presence of rapamycin, Complex \#1 displayed a very strong defect in endonuclease activity $(7.5 \pm 2.7$ and $23.0 \pm 4.3 \%$ nicking at 25 and $50 \mathrm{nM}$ concentrations, respectively), whereas rapamycin had no effect on wild-type protein $(39.0 \pm 3.0$ and
$74.0 \pm 8.6 \%$ nicking at 25 and $50 \mathrm{nM}$ concentrations, respectively).

We were curious why Complex \#1's endonuclease activity but not it's ATPase activity was affected by restricting the Mlh1 IDR (Figure 4B, C and Supplementary Figure S4A). These observations also encouraged us to understand why the mlh1-FRB $355, F K B P_{464}$ allele (forming Complex \#1 in vivo) conferred a weak mutator phenotype in the absence of rapamycin (51.9-fold higher than wild-type; Table 1) and why Complex \#1 showed a weaker PCNA stimulated endonuclease activity compared to wild-type (Figure 4C). One possibility is that Complex \#1 is capable of forming the condensed state but is compromised for the conformational rearrangements that bring the DNA strand to be nicked by Mlh1-Pms1 close to the nuclease active site. To test this, we examined the stimulation of Complex \#1 at different PCNA concentrations. As shown in Figure 4D and Supplementary Figure S4B, we found that the activation of Complex \#1 in the absence of rapamycin was reduced at lower PCNA levels compared to wild-type. For example, at $50 \mathrm{nM}$ PCNA, $58 \%$ of pUC18 DNA was nicked by Mlh1-Pms1 whereas only $20 \%$ was nicked by Complex \#1. These observations indicated that the Mlh1 IDR is important for coordinating conformational rearrangements that bring the nicked DNA strand to the nuclease active site.

\section{DISCUSSION}

In this study, we examined roles for the MLH IDRs in coordinating the recruitment of MLH proteins during MMR. We found that handcuffing the IDRs of Mlh1 and Pms1 through the use of rapamycin-induced FRB-FKBP dimerization disrupted MMR. This phenotype and biochemical analysis of defective complexes provided evidence that the MLH IDRs undergo coordinated intra and inter-subunit interactions that both positively and negatively regulated MLH complex activity $(9,19,20)$.

Our work provides molecular evidence for Mlh1 acting as a 'trigger' to initiate protein-protein and enzymatic functions in MMR (Figure 1A). Disrupting the Mlh1 IDR via intra-subunit FRB-FKBP interactions in Complex \#1 caused a complete defect in MMR and disrupted endonuclease activity (Figure 4). Interestingly Complex \#1 could still bind to DNA in the presence of ATP and rapamycin (though more weakly than wild-type Mlh1-Pms1), consistent with our previous work (23) in which we showed that a deletion of the IDR in Mlh1 conferred a complete defect in MMR but did not dramatically affect the ability of the mutant complex to bind to DNA or interact with Msh2-Msh6. We also saw novel cross-links for Complex 
A

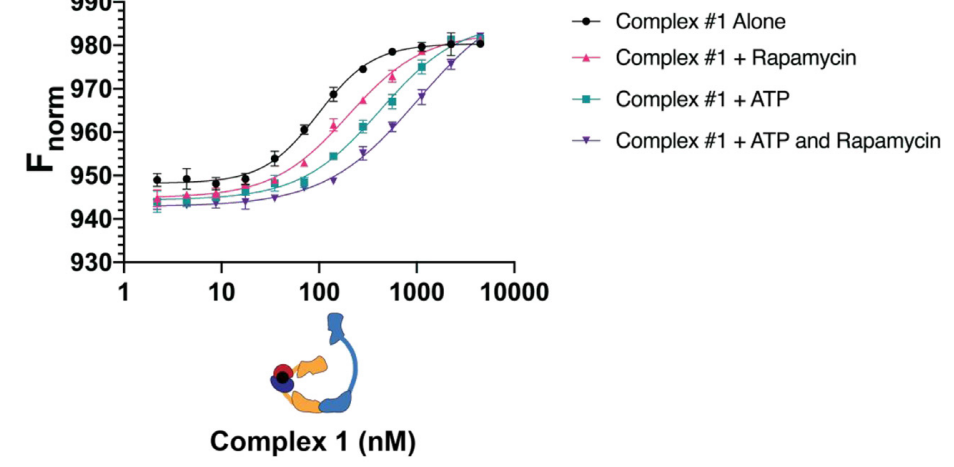

B
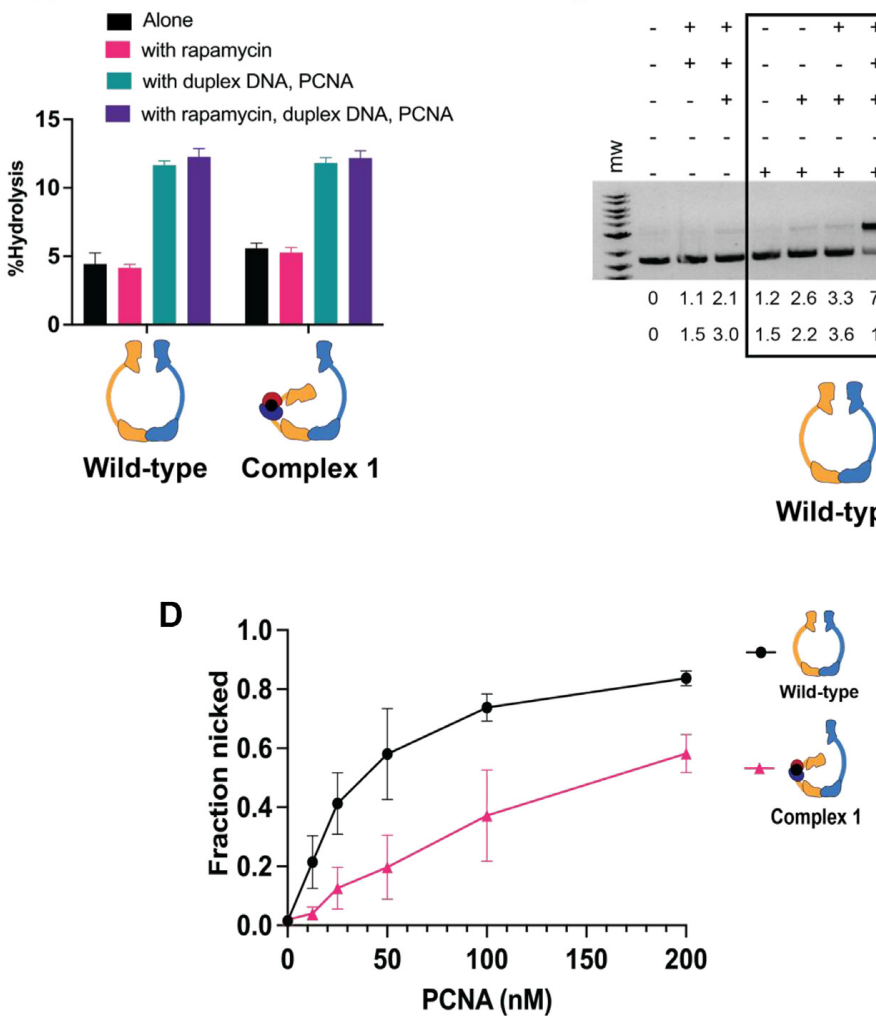

C

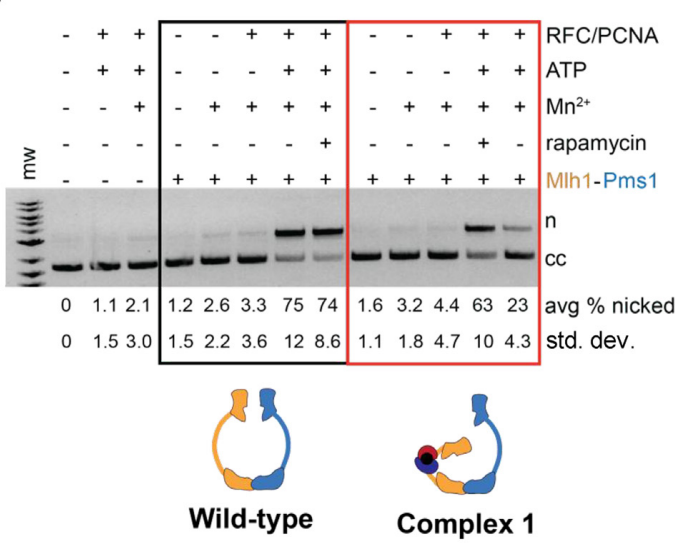

Figure 4. Complex \#1 (mlh1-FRB $355, \mathrm{FKBP}_{464}-\mathrm{Pms} 1$ ) displays defective endonuclease activity in the presence of rapamycin. (A) MST analysis of Complex \#1 in the presence and absence of $49 \mathrm{bp}$ homoduplex DNA $(20 \mathrm{nM})$, ATP $(1 \mathrm{mM})$ and rapamycin $(1 \mu \mathrm{M})$. Three independent experiments (error bars indicate the mean \pm standard deviation) were performed on two independently purified batches of protein. $F_{\text {norm }}$ was calculated as described in Figure $3 \mathrm{~A}$ and the Materials and Methods. See Figure 3A for analysis of Mlh1-Pms1. (B) ATP hydrolysis activities of Mlh1-Pms1 and Complex \#1 (0.40 $\mu$ M each) were determined in the presence and absence of 49-bp homoduplex DNA $(0.75 \mu \mathrm{M})$, PCNA $(0.250 \mu \mathrm{M})$, and rapamycin $(1 \mu \mathrm{M})$. Error bars indicate \pm 1 standard deviation for three replicates. (C) Endonuclease activities of Mlh1-Pms1 and Complex \#1 (50 nM each) determined on a closed circular pUC19 DNA substrate (cc) in the presence (+) or absence $(-)$ of $\mathrm{MnSO}_{4}, \mathrm{ATP}$, rapamycin, and yeast PCNA/RFC (Materials and Methods). MnSO 4 , ATP, rapamycin, RFC and PCNA were included at $5 \mathrm{mM}, 0.5 \mathrm{mM}, 1 \mu \mathrm{M}, 125 \mathrm{nM}$ and $250 \mathrm{nM}$, respectively. $\mathrm{n}=$ nicked product. Error-bars indicate the standard deviation of three replicates (See Supplementary Figure S4A). (D) Endonuclease activities of Mlh1-Pms1 and Complex \#1 (50 nM each) determined on a closed circular DNA substrate (cc) in the presence (+) or absence (-) of $\mathrm{MnSO}_{4}, \mathrm{ATP}$, rapamycin, yeast RFC, and the indicated concentrations of yeast PCNA $\left(12.5,25,50,100,200 \mathrm{nM}\right.$; Materials and Methods). $\mathrm{MnSO}_{4}$, ATP, rapamycin, and RFC were included at $5 \mathrm{mM}, 0.5 \mathrm{mM}, 1 \mu \mathrm{M}$ and $125 \mathrm{nM}$, respectively. $\mathrm{n}=$ nicked product. Error-bars indicate the standard deviation of three replicates (see Supplementary Figure S4B). 
\#5 between the Mlh1 N-terminal domain and the Pms1 IDR, suggesting the presence of a single-arm condensed complex involving the N-terminal domain of Mlh1 (21). Consistent with this observation is the finding that Complex \#5 displayed an increased ATPase and nicking activity in the presence of rapamycin, suggesting that it had been activated in the absence of forming a stable complex on DNA. The idea of Mlh1 serving as a trigger is intriguing because Mlh1 serves as a common subunit for three MutL complexes in baker's yeast, Mlh1-Pms1, Mlh1-Mlh3 and Mlh1-Mlh2, where Mlh1-Mlh3 plays a minor role in MMR and a major role in the resolution of double-Holliday junctions in meiosis to form crossovers, and Mlh1-Mlh2 regulates gene conversion tract lengths in meiosis (reviewed in 59). In such a model, recruitment of various MLHs is accomplished through the initial interactions of Mlh1 with a particular repair pathway, followed by clamp formation by the specificity subunit (Mlh2, Mlh3, or Pms1) that activates the complex for its specific role. Thus, our studies provide mechanistic support for the idea that IDRs license Mlh1-Pms1 to interact stably interact with DNA during MMR. It complements previous studies showing that IDRs regulate how a DNA repair enzyme scans chromatin for DNA lesions and how repair functions (for example, nicking of the newly replicated strand during MMR) are activated $(6,9,19,27,29)$.

How can we explain what we believe is an inappropriate activation of Complex \#5 in the presence of rapamycin? As outlined in the Introduction, the MLH proteins undergo conformational changes throughout the ATP hydrolysis cycle. Studies on Bacillus MutL $(31,60)$ have suggested that ATP-dependent conformational rearrangements bring the DNA strand to be nicked by MLH proteins close to the nuclease active site, and structural and bulk and single molecule analyses of Mlh1-Pms1 have led to a model where ATP hydrolysis by Mlh1-Pms1 returns the complex to an open state where it dissociates from DNA $(11,21,30,60)$. Lastly, studies have suggested in eukaryotes the presence of an alternative Exo1-independent MMR pathway that involves iterative nicking through Mlh1-Pms1 complexes activated via interactions with MSH complexes and PCNA. These observation and work from Kim et al. (9) showing that the Mlh1-Pms1 ATPase activity is disrupted when the IDRs are shortened, suggest a mechanism in which disrupting the MLH conformational cycle through the IDRs impacts MLH ATPase activity. Why is Complex \#5 inappropriately activated in the presence of rapamycin? We hypothesize that the IDRs in Complex \#5 in the presence of rapamycin are forced into an inappropriate conformational state that promotes complex dissociation and reiterative nicking of DNA in the absence of a stable clamp state required in MMR (27). Such an explanation, which is consistent with the MMR defect seen in strains containing Complex \#5 grown in the presence of rapamycin, will need to be tested in single molecule studies $(6,27)$.

\section{Modulation of MMR using a small molecule}

We used an inducible dimerization approach to modulate MMR functions through the addition and removal of the small molecule rapamycin (33). The reversibility of this method can provide a valuable means to understand how an increased reversion rate can provide beneficial mutations to an organism adapting to changing environments (reviewed in 61,62). Elevated mutation rates, while providing a source of beneficial mutations for adaptation, are associated with long-term fitness costs due to the accumulation of deleterious mutations (63-65). In the lys2- $A_{14}$ reversion assay where the difference between wild-type and MMR defective is roughly 5000 -fold, we identified complexes that showed wild-type or near wild-type phenotypes in the absence of rapamycin, but a wide-range of elevated reversion rates in its presence (Table 1). For example, in the presence of rapamycin, Complex \#2 conferred a 79.5-fold elevated rate compared to wild-type, Complex \#3, a 984-fold higher rate, Complex \#5, a 1440-fold higher rate, and Complex \#1, a rate indistinguishable from $\mathrm{mlhl}$ null (5190-fold). Most informative was that the null phenotype in Complex \#1 could be reversed to the level observed in the absence of rapamycin. This resource can thus allow one to grow cells in stressed environments (such as high salt) and then pulse them with rapamycin for different time intervals under a variety of mutation supply conditions. Such experiments would allow one to determine the impact of cellular fitness as a function of mutational load and would be relevant to understand the progression of disease states accelerated by increased mutation rate.

Mlh1-Mlh3 has been shown in meiosis in both lower and higher eukaryotes to facilitate the resolution of doubleHolliday junction intermediates into crossovers. These crossovers facilitate the segregation of homologs in the first meiotic division (reviewed in 66). The use of a small molecule to potentially modulate the MMR and crossover resolution functions of Mlh1-Mlh3 at different stages in meiosis could thus be valuable in teasing apart proposed separable functions for MLH proteins in meiosis (67).

\section{An application of the FRB-FKBP dimerization system}

IDRs are characterized as being highly dynamic and without well-defined structures. However, many IDRs have been shown to undergo disorder to order transitions, and in some cases form different structures when complexed with different partners (reviewed in 2,3). Interestingly a length distribution analysis of human proteins obtained from the SwissProt data base indicated that very long IDRs (>500 amino acids) were found in higher numbers than predicted (68). Furthermore, gene ontology analysis indicated that IDRs greater than 500 amino acids in length are overrepresented in transcription functions and those between 300 and 500 amino acids in length are enriched in kinase and phosphatase functions (69). Thus, our strategy to introduce FRB and FKBP domains into the IDRs of the MLH proteins could be applied to reversibly block or lock in specific conformational states of IDRs that regulate a variety of cellular processes.

\section{DATA AVAILABILITY}

This study includes no data deposited in external repositories. 


\section{SUPPLEMENTARY DATA}

Supplementary Data are available at NAR Online.

\section{ACKNOWLEDGEMENTS}

We are grateful to Eric Greene for suggesting the use of the FRB-FKBP dimerization to probe Mlh1-Pms1 functions, and Ilya Finkelstein, Carol Manhart, Joshua Chappie, Philip Cole, Sy Redding, Myfanwy Adams, Fred Dyda, Alison Hickman, and Jeff Jorgensen for providing their expertise, advice, and use of equipment. C.M.F. performed the experiments described in Figures 3, 4, Supplementary Figures S1-S4, and Tables 1-3. C.M.F. performed the DSSO cross linking for the MLH complexes presented in Figure 2 and Supplementary Table S3, with the mass-spectrometry analyses performed by T.-Y.W., Q.Z. and K.Y.. C.M.F., T.Y.W., Q.Z. and E.A. wrote the initial draft of the manuscript and all authors participated in preparing and revising the manuscript. The content of this study is solely the responsibility of the authors and does not necessarily represent the official views of the National Institutes of Health. The funders had no role in study design, data collection and analysis, decision to publish, or preparation of the manuscript.

\section{FUNDING}

National Institute of General Medical Sciences of the National Institutes of Health (https://www.nih. gov/) [R35GM134872 to C.M.F. and E.A., R01GM124559 to T.-Y.W., Q.Z., K.Y., H.Y.]. Funding for open access charge: NIH.

Conflict of interest statement. None declared.

\section{REFERENCES}

1. Oldfield,C.J. and Dunker,A.K. (2014) Intrinsically disordered proteins and intrinsically disordered protein regions. Ann. Rev. Biochem., 83, 553-584.

2. Van Der Lee,R., Buljan,M., Lang,B., Weatheritt,R.J., Daughdrill,G.W., Dunker,A.K., Fuxreiter,M., Gough,J., Gsponer,J., Jones,D.T. et al. (2014) Classification of intrinsically disordered regions and proteins. Chem. Rev., 114, 6589-6631.

3. Uversky,V.N. (2019) Intrinsically disordered proteins and their "mysterious" (meta) physics. Front. Phys. , 7, 10.

4. Kunkel,T.A. and Erie,D.A. (2015) Eukaryotic mismatch repair in relation to DNA replication. Ann. Rev. Genet., 49, 291-313.

5. Gradia,S., Subramanian,D., Wilson,T., Acharya,S., Makhov,A., Griffith,J. and Fishel,R. (1999) hMSH2-hMSH6 forms a hydrolysis-independent sliding clamp on mismatched DNA. Mol. Cell, 3, 255-261.

6. Gorman,J., Wang,F., Redding,S., Plys,A.J., Fazio,T., Wind,S., Alani,E.E. and Greene,E.C. (2012) Single-molecule imaging reveals target-search mechanisms during DNA mismatch repair. Proc. Natl. Acad. Sci. U.S.A., 109, E3074-E3083.

7. Pluciennik,A., Dzantiev,L., Iyer,R.R., Constantin,N., Kadyrov,F.A. and Modrich,P. (2010) PCNA function in the activation and strand direction of MutL $\alpha$ endonuclease in mismatch repair. Proc. Natl. Acad. Sci. U.S.A., 107, 16066-16071.

8. Kawasoe,Y., Tsurimoto,T., Nakagawa,T., Masukata,H. and Takahashi,T.S. (2016) MutS $\alpha$ maintains the mismatch repair capability by inhibiting PCNA unloading. Elife, $\mathbf{5}$, e15155.

9. Kim,Y., Furman,C.M., Manhart,C.M., Alani,E. and Finkelstein,I.J. (2019) Intrinsically disordered regions regulate both catalytic and non-catalytic activities of the MutL $\alpha$ mismatch repair complex. Nucleic Acids Res. , 47, 1823-1835.
10. Kadyrov,F.A., Dzantiev,L., Constantin,N. and Modrich,P. (2006) Endonucleolytic function of MutLalpha in human mismatch repair. Cell, 126, 297-308.

11. Tran,P.T. and Liskay,R.M. (2000) Functional studies on the candidate ATPase domains of Saccharomyces cerevisiae. MutL $\alpha$. Mol. Cell. Biol., 20, 6390-6398.

12. Räschle,M., Dufner,P., Marra,G. and Jiricny,J. (2002) Mutations within the hMLH1 and hPMS2 subunits of the human MutL $\alpha$ mismatch repair factor affect its ATPase activity, but not its ability to interact with hMutS $\alpha$. J. Biol. Chem., 277, 21810-21820.

13. Genschel,J., Kadyrova,L.Y., Iyer,R.R., Dahal,B.K., Kadyrov,F.A. and Modrich,P. (2017) Interaction of proliferating cell nuclear antigen with PMS2 is required for MutL $\alpha$ activation and function in mismatch repair. Proc. Natl. Acad. Sci. U.S.A., 114, 4930-4935.

14. Bowen,N. and Kolodner,R.D. (2017) Reconstitution of Saccharomyces cerevisiae DNA polymerase $\varepsilon$-dependent mismatch repair with purified proteins. Proc. Natl. Acad. Sci. U.S.A., 114, $3607-3612$.

15. Kadyrov,F.A., Genschel,J., Fang,Y., Penland,E., Edelmann,W. and Modrich,P. (2009) A possible mechanism for exonuclease 1-independent eukaryotic mismatch repair. Proc. Natl. Acad. Sci. U.S.A., 106, 8495-8500.

16. Goellner,E.M., Smith,C.E., Campbell,C.S., Hombauer,H., Desai,A., Putnam,C.D. and Kolodner,R.D. (2014) PCNA and Msh2-Msh6 activate an Mlh1-Pms1 endonuclease pathway required for Exo1-independent mismatch repair. Mol. Cell, 55, 291-304.

17. Goellner,E.M., Putnam,C.D. and Kolodner,R.D. (2015) Exonuclease 1-dependent and independent mismatch repair. DNA Repair (Amst.), 32, 24-32.

18. Hermans, N., Laffeber,C., Cristovao,M., Artola-Borán,M., Mardenborough,Y., Ikpa,P., Jaddoe,A, Winterwerp,H.H., Wyman,C., Jiricny,J. et al. (2016) Dual daughter strand incision is processive and increases the efficiency of DNA mismatch repair. Nucleic Acids Res., 44, 6770-6786.

19. Gorman,J., Plys,A.J., Visnapuu,M.L., Alani,E. and Greene,E.C. (2010) Visualizing one-dimensional diffusion of eukaryotic DNA repair factors along a chromatin lattice. Nat. Struct. Mol. Biol., 17, 932-938.

20. Hall,M.C., Shcherbakova,P.V., Fortune,J.M., Borchers,C.H., Dial,J.M., Tomer,K.B. and Kunkel,T.A. (2003) DNA binding by yeast Mlh1 and Pms1: implications for DNA mismatch repair. Nucleic Acids Res., 31, 2025-2034.

21. Sacho,E.J., Kadyrov,F.A., Modrich,P., Kunkel,T.A. and Erie,D.A. (2008) Direct visualization of asymmetric adenine nucleotide-induced conformational changes in MutL $\alpha$. Mol. Cell, 29, 112-121.

22. Hall,M.C., Wang,H., Erie,D.A. and Kunkel,T.A. (2001) High affinity cooperative DNA binding by the yeast Mlh1-Pms1 heterodimer. $J$. Mol. Biol., 312, 637-647.

23. Plys,A.J., Rogacheva,M.V., Greene,E.C. and Alani,E. (2012) The unstructured linker arms of Mlh1-Pms1 are important for interactions with DNA during mismatch repair. J. Mol. Biol., 422, 192-203.

24. Prolla,T.A., Christie,D.M. and Liskay,R.M. (1994) Dual requirement in yeast DNA mismatch repair for $M L H 1$ and $P M S 1$, two homologs of the bacterial mutL gene. Mol. Cell. Biol., 14, 407-415.

25. Argueso,J.L., Kijas,A.W., Sarin,S., Heck,J., Waase,M. and Alani,E. (2003) Systematic mutagenesis of the Saccharomyces cerevisiae MLH1 gene reveals distinct roles for Mlhlp in meiotic crossing over and in vegetative and meiotic mismatch repair. Mol. Cell. Biol., 23, 873-886.

26. Guarné,A., Ramon-Maiques,S., Wolff,E.M., Ghirlando,R., Hu,X., Miller,J.H. and Yang,W. (2004) Structure of the MutL C-terminal domain: a model of intact MutL and its roles in mismatch repair. EMBO J., 23, 4134-4145.

27. Liu,J., Hanne,J., Britton,B.M., Bennett,J., Kim,D., Lee,J.B. and Fishel,R. (2016) Cascading MutS and MutL sliding clamps control DNA diffusion to activate mismatch repair. Nature, 539, 583-587.

28. Groothuizen,F.S., Winkler,I., Cristóvão,M., Fish,A., Winterwerp,H.H., Reumer,A., Marx,A.D., Hermans,N., Nicholls,R.A., Murshudov,G.N. et al. (2015) MutS/MutL crystal structure reveals that the MutS sliding clamp loads MutL onto DNA. eLife, 4, e06744.

29. Mardenborough,Y.S., Nitsenko,K., Laffeber,C., Duboc,C., Sahin,E., Quessada-Vial,A., Winterwerp,H.H., Sixma,T.K., Kanaar,R., Friedhoff,P. et al. (2019) The unstructured linker arms of MutL 
enable GATC site incision beyond roadblocks during initiation of DNA mismatch repair. Nucleic Acids Res., 47, 11667-11680.

30. Ban,C, Junop,M. and Yang,W. (1999) Transformation of MutL by ATP binding and hydrolysis, a switch in DNA mismatch repair. Cell, 97, 85-97.

31. Pillon,M.C., Lorenowicz,J.J., Uckelmann,M., Klocko,A.D., Mitchell,R.R., Chung,Y.S., Modrich,P, Walker,G.C., Simmons,L.A., Friedhoff,P. et al. (2010) Structure of the endonuclease domain of MutL, Unlicensed to cut. Mol. Cell, 39, 145-151.

32. Gueneau,E., Dherin,C., Legrand,P., Tellier-Lebegue,C., Gilquin,B., Bonnesoeur,P., Londino,F., Quemener,C., Le Du,M.H., Márquez,J.A. et al. (2013) Structure of the MutL $\alpha$ C-terminal domain reveals how Mlh1 contributes to Pms1 endonuclease site. Nat. Struct. Mol. Biol., 20, 461-468.

33. Spencer,D.M., Wandless,T.J., Schreiber,S.L. and Crabtree,G.R. (1993) Controlling signal transduction with synthetic ligands. Science, 262, 1019-1024

34. Rose,M.D., Winston,F. and Hieter,P. (1990) In: Laboratory Course Manual for Methods in Yeast Genetics. Cold Spring Harbor Laboratory Press, NY

35. Goldstein,A.L. and McCusker,J.H. (1999) Three new dominant drug resistance cassettes for gene disruption in Saccharomyces cerevisiae. Yeast, 15, 1541-1553.

36. Zhu,L., Jorgensen,J.R., Li,M., Chuang,Y.S. and Emr,S.D. (2017) ESCRTs function directly on the lysosome membrane to downregulate ubiquitinated lysosomal membrane proteins. eLife, $\mathbf{6}$, e26403.

37. Gietz,R.D., Schiestl,R.H., Willems,A.R. and Woods,R.A. (1995) Studies on the transformation of intact yeast cells by the LiAc/SS-DNA/PEG procedure. Yeast, 11, 355-360.

38. Xu,T., Johnson,C.A., Gestwicki,J.E. and Kumar,A. (2010) Conditionally controlling nuclear trafficking in yeast by chemical-induced protein dimerization. Nat. Protoc., 5, 1831-1843.

39. Geda,P., Patury,S., Ma,J., Bharucha,N., Dobry,C.J., Lawson,S.K., Gestwicki,J.E. and Kumar,A. (2008) A small molecule-directed approach to control protein localization and function. Yeast, 25, 577-594.

40. Christianson,T.W., Sikorski,R.S., Dante,M., Shero,J.H. and Hieter,P. (1992) Multifunctional yeast high-copy-number shuttle vectors. Gene, 110, 119-122.

41. Hall,M.C. and Kunkel,T.A. (2001) Purification of eukaryotic MutL homologs from Saccharomyces cerevisiae using self-cleaving affinity technology. Prot. Exp. Purif., 21, 333-342.

42. Tran,H.T., Keen,J.D., Kricker,M., Resnick,M.A. and Gordenin,D.A (1997) Hypermutability of homonucleotide runs in mismatch repair and DNA polymerase proofreading yeast mutants. Mol. Cell. Biol., 17, 2859-2865.

43. Dixon,W.J. and Massey,F.J. (1969) In: Introduction to Statistical Analysis. McGraw-Hill, NY.

44. Yugandhar,K., Wang,T.Y., Leung,A.K.Y., Lanz,M.C., Motorykin,I., Liang,J., Shayhidin,E.E., Smolka,M.B., Zhang,S. and Yu,H. (2020) MaXLinker, proteome-wide cross-link identifications with high specificity and sensitivity. Mol. Cell Proteomics, 19, 554-568.

45. Cooper,M.P., Balajee,A.S. and Bohr,V.A. (1999) The C-terminal domain of p21 inhibits nucleotide excision repair in vitro and in vivo. Mol. Biol. Cell, 10, 2119-2129.

46. Finkelstein,J., Antony,E., Hingorani,M.M. and O’Donnell,M. (2003) Overproduction and analysis of eukaryotic multiprotein complexes in Escherichia coli using a dual-vector strategy. Anal. Biochem., 319, 78-87.

47. Rogacheva,M.V., Manhart,C.M., Chen,C., Guarne,A., Surtees,J. and Alani,E. (2014) Mlh1-Mlh3, a meiotic crossover and DNA mismatch repair factor, is a Msh2-Msh3-stimulated endonuclease. J. Biol. Chem., 289, 5664-5673.

48. Hosford,C.J., Adams,M.C., Niu,Y. and Chappie,J.S. (2020) The N-terminal domain of Staphylothermus marinus McrB shares structural homology with PUA-like RNA binding proteins. J. Struct. Biol., 211, 107572.
49. Yu,C. and Huang,L. (2018) Cross-linking mass spectrometry: an emerging technology for interactomics and structural biology. Anal. Chem., 90, 144-165.

50. Smith,C.E., Mendillo,M.L., Bowen,N., Hombauer,H., Campbell,C.S., Desai,A., Putnam,C.D. and Kolodner,R.D. (2013) Dominant mutations in S cerevisiae PMS1 identify the Mlh1-Pms1 endonuclease active site and an exonuclease 1-independent mismatch repair pathway. PLos Genet., 9, e1003869.

51. Voß,S., Klewer,L. and Wu,Y.W. (2015) Chemically induced dimerization: reversible and spatiotemporal control of protein function in cells. Curr. Opin. Chem. Biol., 28, 194-201.

52. Inobe,T. and Nukina,N. (2016) Rapamycin-induced oligomer formation system of FRB-FKBP fusion proteins. J. Biosci. Bioeng. 122, 40-46.

53. Gruber,S., Arumugam,P., Katou,Y., Kuglitsch,D., Helmhart,W., Shirahige,K. and Nasmyth,K. (2006) Evidence that loading of cohesion onto chromosomes involves opening of its SMC hinge. Cell, 127, 523-537.

54. Banaszynski,L.A., Liu,C.W. and Wandless,T.J. (2005) Characterization of the FKBP $\odot$ Rapamycin $\odot$ FRB ternary complex. J. Am. Chem. Soc., 127, 4715-4721.

55. Fegan,A., White,B., Carlson,J.C. and Wagner,C.R. (2010) Chemically controlled protein assembly, techniques and applications. Chem, Rev, 110, 3315-3336.

56. Ho,B., Baryshnikova,A. and Brown,G.W. (2018) Unification of protein abundance datasets yields a quantitative Saccharomyces cerevisiae proteome. Cell Syst., 6, 192-205.

57. Jorgensen,P., Edgington,N.P., Schneider,B.L., Rupes,I., Tyers,M. and Futcher,B. (2007) The size of the nucleus increases as yeast cells grow. Mol. Biol. Cell, 18, 3225-3709.

58. Kadyrov,F.A., Holmes,S.F., Arana,M.E., Lukianova,O.A., O’Donnell,M., Kunkel,T.A. and Modrich,P. (2007) Saccharomyces cerevisiae MutL $\alpha$ is a mismatch repair endonuclease. J. Biol. Chem., 282, 37181-37190.

59. Furman,C.M., Elbashir,R. and Alani,E. (2021) Expanded roles for the MutL family of DNA mismatch repair proteins. Yeast, 38, 39-53.

60. Ban,C. and Yang,W. (1998) Crystal structure and ATPase activity of MutL: implications for DNA repair and mutagenesis. Cell, 95, 541-552.

61. Raghavan,V., Aquadro,C.F. and Alani,E. (2019) Baker's yeast clinical isolates provide a model for how pathogenic yeasts adapt to stress Trends Genet., 35, 804-817.

62. Raghavan,V., Bui,D.T., Al-Sweel,N., Friedrich,A., Schacherer,J. Aquadro,C.F. and Alani,E. (2018) Incompatibilities in mismatch repair genes $M L H 1-P M S 1$ contribute to a wide range of mutation rates in human isolates of baker's yeast. Genetics, 210, 1253-1266.

63. Lynch,M., Ackerman,M.S., Gout,J.F., Long,H., Sung,W., Thomas,W.K. and Foster,P.L. (2016) Genetic drift, selection and the evolution of the mutation rate. Nat. Rev. Genet., 17, 704.

64. Bui,D.T., Dine,E., Anderson,J.B., Aquadro,C.F. and Alani,E.E. (2015) A genetic incompatibility accelerates adaptation in yeast. PLoS Genet., 11, e1005407.

65. Bui,D.T., Friedrich,A., Al-Sweel,N., Liti,G., Schacherer,J., Aquadro,C.F. and Alani,E. (2017) Mismatch repair incompatibilities in diverse yeast populations. Genetics, 205, 1459-1471.

66. Manhart,C.M. and Alani,E. (2016) Roles for mismatch repair family proteins in promoting meiotic crossing over. DNA Repair (Amst.), 38, 84-93.

67. Claeys Bouuaert,C. and Keeney,S. (2017) Distinct DNA-binding surfaces in the ATPase and linker domains of MutL $\gamma$ determine its substrate specificities and exert separable functions in meiotic recombination and mismatch repair. PLos Genet., 13, e1006722.

68. Tompa,P. and Kalmar,L. (2010) Power law distribution defines structural disorder as a structural element directly linked with function. J. Mol. Biol., 403, 346-350.

69. Lobley,A., Swindells,M.B., Orengo,C.A. and Jones,D.T. (2007) Inferring function using patterns of native disorder in proteins. PLoS Comp. Biol., 3, e162. 